NANO: Brief Reports and Reviews

Vol. 14, No. 4 (2019) 1930004 (22 pages)

(C) World Scientific Publishing Company

DOI: 10.1142/S1793292019300044

\title{
Review of the Preparation and Structures of Si Nanowires, Ge Quantum Dots and Their Composites
}

\author{
Xiaokang Weng \\ School of Materials Science and Engineering \\ Yunnan Key Laboratory for the \\ Micro/Nano Materials and Technology \\ Yunnan University, Kunming 650091, P. R. China \\ xkw@mail.ynu.edu.cn
}

Jie Yang*, Dongze Li, Rongfei Wang, Feng Qiu, Chong Wang ${ }^{\dagger}$ and Yu Yang

School of Materials Science and Engineering

Yunnan Key Laboratory for the

Micro/Nano Materials and Technology

School of Energy, Yunnan University

Kunming 650091, P. R. China

*jieyang@ynu.edu.cn

†cwang@ynu.edu.cn

Received 13 November 2018

Accepted 28 February 2019

Published 3 April 2019

\begin{abstract}
Because the motion of charge carriers in nanowires and quantum dots is restricted within nanoscale in two and three dimensions, respectively, both nanowires and quantum dots exhibit many excellent optoelectronic properties. Particularly, with the advantage of being compatible with Si integrated circuits, Silicon nanowires (SiNWs) and germanium quantum dots (GeQDs) have been extensively studied in the past few decades. In order to explore novel physical properties, the integration of SiNWs and GeQDs has attracted great attention recently. In this paper, recent researches on the preparation methods and structures of SiNWs, GeQDs and their composites are reviewed, respectively. The synthesis of SiNWs with random distribution and ordered arrays by using vaporliquid-solid growth mechanism and metal-assisted chemical etching technique is firstly summarized. Some special structures of SiNWs are also discussed. Furthermore, the development of some novel structures of GeQDs for further improving their optical properties is reviewed. Finally, the growth mechanism and structure evolution of SiNWs/GeQDs composites are illustrated from the view of theory and experiment. The strain in Ge shell layers and SiNWs, the relationship between Ge growth mode and SiNW diameter, and the distribution of GeQDs on the radial and axial directions of SiNWs are discussed in detail. The research about the growth of SiNWs/GeQDs composite structures is in its early stage, so there are many questions that need to be resolved in future.
\end{abstract}

Keywords: Silicon nanowire; Ge quantum dot; composite structure; core/shell nanowire; ordered array.

${ }^{*}$ Corresponding author. 


\section{Introduction}

With the development of nanotechnology, there are increasing demands for smaller and more efficient nanodevices. However, the properties of nanomaterials comprised of a single structure or substance are often unsuitable for such applications. Therefore, many novel nanocomposite materials have been proposed, such as porous nanowires, ${ }^{1}$ coreshell nanowires, ${ }^{2,3}$ and carbon nanotube composite materials. ${ }^{4,5}$ In particular, silicon nanowires (SiNWs) are promising potential materials for future nano-optoelectronic information applications due to silicon's important role in the traditional microelectronic and photovoltaic fields. Recently, $\mathrm{Si} / \mathrm{Ge}$ core-shell nanowire composites have attracted great attention due to their superior performance. Instead of Ge nanofilm layer, the integration of germanium quantum dots (GeQDs) and SiNWs can combine the characteristics of one-dimensional and zero-dimensional nanomaterials, which have promising applications in nanoscale light-emitting diodes,${ }^{6}$ photoelectric detectors,${ }^{7,8}$ solar cells, ${ }^{9}$ fieldeffect transistors ${ }^{10}$ (FET) and thermoelectric energy conversion, ${ }^{11}$ because of the stronger quantum confinement effect of GeQDs. Fortunately, a comprehensive understanding of the preparation, structure and performance of SiNWs and GeQDs has been gained after several decades of research and exploration, laying a good foundation for the study of SiNW/GeQD composite materials.

Currently, some research progress in SiNW/ GeQD composite materials has been achieved. Fukata et al. ${ }^{12}$ pointed out that, theoretically, the critical thickness of the shell layer for transition of Ge growth mode increases with decrease in the nanowire diameter. The smaller the nanowire diameter, the easier it is for $\mathrm{Si} / \mathrm{Ge}$ core-shell nanowire structures to form. Li et al. ${ }^{13}$ proposed a physical model to interpret the evolution of Ge layer growth on the radial surface of SiNWs with increasing nanowire diameter. They demonstrated that the curved surface of the nanowire is conducive to the release of misfit strain. The growth of GeQDs on the radial surfaces of SiNWs was studied experimentally by Pan and Kwon et al..$^{2,14}$ under various conditions. However, their results concerning the critical diameter of SiNWs for the transition of Ge growth mode were inconsistent. This indicates that the growth mechanism of GeQDs on the radial surfaces of SiNWs still needs to be further researched. For the study of electric properties of SiNW/GeQD composites, Dhungana et al. ${ }^{15}$ confirmed that transconductance of SiNW/GeQD FET is substantially higher than that of SiNW FET, which suggests much higher carrier mobility in the SiNW/GeQD device.

In order to illustrate the research progress about SiNW/GeQD composites clearly in this review, we firstly summarize the recent research advances in the preparation and structures of SiNWs and GeQDs. Then, the growth mechanism and structure evolution of that composites are reviewed from the view of theory and experiment. Some potential issues and possible development directions of that composites for further research are discussed.

\section{Synthesis and Structures of SiNWs}

In 1964, silicon whiskers were successfully synthesized by the vapor-liquid-solid (VLS) method, ${ }^{16}$ which set the basis for the synthesis of SiNWs. In the mid-1990s, abundant SiNWs with nanoscopic dimensions were prepared by laser ablation (LA) techniques, ${ }^{17}$ which triggered great interest in SiNW research. After nearly 30 years of development, SiNWs can be fabricated by bottom-up and top-down approaches. Top-down fabrication methods mainly include various dry and wet etching techniques. Bottom-up approaches principally include LA and chemical vapor deposition (CVD). As shown in Table 1, recent research progress on the preparation and structures of SiNWs has made the following three main achievements: (1) the arrangement of SiNWs can be controlled from a random distribution to an ordered array. The diameter of SiNWs in an ordered array can be controlled at about $10 \mathrm{~nm} .{ }^{18}(2)$ SiNWs with small diameters and large aspect ratios have been prepared. (3) SiNWs with various structures have been produced, such as porous nanowires, $p$ - $n$ nanowire superlattices, tapered nanowires, ultra-thin structures, kinked structures and zigzag structures. Additionally, SiNWs' electrical properties (such as field emission characteristics, ${ }^{19}$ single electron effects ${ }^{20}$ and Coulomb blockade effects ${ }^{21}$ ), photoluminescence ${ }^{22}$ and photoelectric properties (such as photoelectric conversion. ${ }^{23}$ and photoelectric detection ${ }^{24}$ ) have been studied extensively. 
Table 1. Main preparation methods and structural characteristics of Si nanowires in recent years.

\begin{tabular}{|c|c|c|c|c|c|c|c|c|}
\hline Method & Year & Ordered arrays & Template & $\begin{array}{l}\text { Diameter } \\
(\mathrm{nm})\end{array}$ & $\begin{array}{l}\text { Height } \\
(\mu \mathrm{m})\end{array}$ & $\begin{array}{c}\text { Aspect } \\
\text { ratio }\end{array}$ & Structural characteristics & Ref. \\
\hline \multirow[t]{4}{*}{ VLS } & 1964 & $\mathrm{~N}$ & / & 100 & / & / & Whiskers & 16 \\
\hline & 1998 & $\mathrm{~N}$ & / & $6-20$ & $1-30$ & 166 & NWs & 25 \\
\hline & 2004 & $\mathrm{~N}$ & / & $3-30$ & / & / & NWs & 26 \\
\hline & 2017 & $\mathrm{~N}$ & / & $10-76$ & 60 & / & $P-n$ NW superlattices & 27 \\
\hline \multirow[t]{2}{*}{ Template + VLS } & 2005 & $\mathrm{Y}$ & NSL & $70-90$ & 0.25 & 3 & NWs & 28 \\
\hline & 2016 & $\mathrm{Y}$ & KSL & 220 & 17 & 77 & NWs & 29 \\
\hline \multirow[t]{4}{*}{ MACE } & 2008 & $\mathrm{~N}$ & / & $20-300$ & $5-150$ & $250-500$ & NWs & 30 \\
\hline & 2009 & $\mathrm{~N}$ & / & $100-200$ & 50 & $250-500$ & Porous NWs & 31 \\
\hline & 2011 & $\mathrm{~N}$ & / & $66-115$ & 5.25 & 46 & Tapered NWs & 32 \\
\hline & 2015 & $\mathrm{~N}$ & / & $6-12$ & 9.6 & $800-1600$ & Ultra-thin NWs & 33 \\
\hline \multirow[t]{7}{*}{ Template + MACE } & 2007 & $\mathrm{Y}$ & NSL & 180 & 5.3 & 30 & NWs & 34 \\
\hline & 2008 & $\mathrm{Y}$ & $\mathrm{AAO}$ & $8-20$ & 0.05 & $2.5-5$ & NWs & 18 \\
\hline & 2009 & $\mathrm{Y}$ & $\mathrm{BC}$ & 20 & 4.4 & 220 & NWs & 35 \\
\hline & 2010 & $\mathrm{Y}$ & LIL & 550 & 100 & 182 & Pillars & 36 \\
\hline & 2011 & $\mathrm{Y}$ & $\mathrm{AAO}$ & $35-73$ & / & / & Smooth NWs & 37 \\
\hline & 2016 & $\mathrm{Y}$ & NSL & Sub-20 & / & / & NWs & 38 \\
\hline & 2017 & $\mathrm{Y}$ & NSL & $100-550$ & $4-35$ & $8-120$ & $\begin{array}{l}\text { Pillars, kinked } \\
\text { and zigzag NWs }\end{array}$ & $39-41$ \\
\hline
\end{tabular}

Notes: Y stands for Yes and N stands for No.

\subsection{SiNWs in a random distribution}

Currently, metal catalysis is one of the major methods used to grow or etch SiNWs. When the positions of metal particles or thin films distributed on the surface of a $\mathrm{Si}$ substrate are not limited, $\mathrm{Si}$ nanowires can form randomly.

In metal catalysis-induced growth methods, SiNWs have been synthesized from bottom to top mainly by CVD, MBE and LA techniques based on the VLS growth method. ${ }^{43}$ The name VLS method refers to the fact that silicon from vapor passes through a liquid droplet and finally ends up as a solid. Many metals, such as $\mathrm{Au}, \mathrm{Cu}, \mathrm{In}, \mathrm{Ga}, \mathrm{Ni}, \mathrm{Pt}$ and $\mathrm{Ti}_{1}{ }^{44,45}$ can catalyze a gaseous silicon precursor (e.g., $\mathrm{SiH}_{4}, \mathrm{Si}_{2} \mathrm{H}_{6}$ or $\mathrm{SiCl}_{4}$ ) to form SiNWs under certain temperatures. The VLS growth process has two stages: firstly, small liquid metal-Si alloy droplets form on the substrate surface. Secondly, the silicon supplied by the gaseous silicon precursor causes the droplet to become supersaturated with $\mathrm{Si}$ until silicon freezes out. This process is schematically presented in Fig. 1. The diameter of the SiNWs

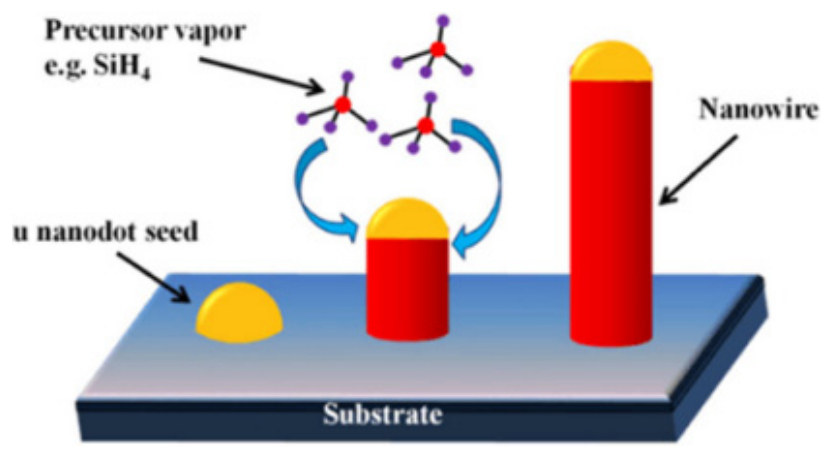

(a)

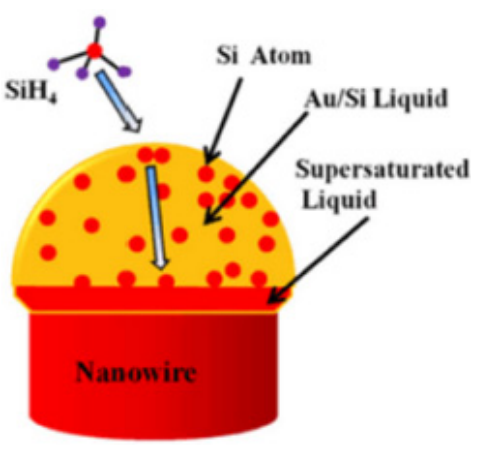

(b)

Fig. 1. Vapor-liquid-solid mechanism for nanowire synthesis, (a) schematic of VLS growth of Si NW from a liquid Au catalyst seed, which floats on top of the NW as it grows. (b) Enlarged view illustrating the kinetic steps for NW growth. Reprinted with permission from Ref. 42. Copyright 2010 Chemical Reviews. 
is determined by the size of the metal-Si, their length is decided by the flow velocity and reaction time.

One of the advantages of VLS growth is that the diameter of SiNWs can be controlled below $10 \mathrm{~nm}$. Based on the VLS growth method, Wu et al. ${ }^{26}$ prepared monocrystalline SiNWs with diameters of 3-30 nm using CVD technique. They demonstrated that the growth directions of SiNWs are dependent on their sizes. The smallest diameter SiNWs grew along $\langle 110\rangle$ direction, while the larger SiNWs grew along $\langle 111\rangle$ direction. SiNWs with a diameter of $10 \mathrm{~nm}$ and length $>1 \mu \mathrm{m}$ were also obtained by the LA technique. ${ }^{46}$

The VLS growth mechanism also allows for precise control of the composition and doping of SiNWs at nanoscale, which is a benefit for fabricating SiNW device with $p$ - $n$ junction. ${ }^{5,27,47}$ Usually, $p$ - and $n$-type doping of SiNWs can be performed during the VLS growth process using CVD by introducing the diborane and phosphine, respectively, in addition to the silane. Compared with the phosphorus doping of SiNWs, there are many problems in boron doping of SiNWs, such as morphological changes or amorphous shell overgrowth. ${ }^{27}$ Because boron can serve to catalyze the decomposition of silane $\left(\mathrm{SiH}_{4}\right)$ under proper conditions, resulting in the increasement of radial growth rate and overgrowth of a $\mathrm{Si}$ shell on nanowire sidewall. The sidewall becomes rough due to the gold-cluster migration from the main catalyst during the overgrowth process. Hydrogen chloride $(\mathrm{HCl})$ was employed to improve the surface morphology of $p$ - and $n$-doped SiNWs. The SiNWs with smoother surface and reduced tapering were observed by Gentile et al. ${ }^{47}$ after using the $\mathrm{HCl}$ with dopant gases, which was attributed to the limitation of gold diffusion from the catalyst. This approach provides a valuable solution for limiting the gold contamination in SiNWs. The gold contamination is harmful for the performance of nanowire device, because it will create traps and reduce minority carrier lifetimes. ${ }^{48}$

Recently, Hill et al. ${ }^{27}$ explored the effect of temperature and $\mathrm{HCl}: \mathrm{SiH}_{4}$ ratio on the $p$-type $\mathrm{SiNW}$ growth. As shown in Fig. 2, as the $\mathrm{HCl}$ flow is low and the temperature is below $700^{\circ} \mathrm{C}$ in region $\mathrm{I}$, the overgrowth of Si shell occurs, leading to the appearance of tapered SiNWs with bases thicker than the original catalyst diameter. In region III, higher $\mathrm{HCl}$ flow over the same temperature regime, SiNW grows slowly. Between these two regimes is region II, where reliable SiNW nucleation and stable growth is achieved. In region IV, unstable SiNW growth is observed. The temperature-dependent kinetics of Si chlorination provide a basis for understanding the origin of the different growth regimes.

Metal-assisted chemical etching (MACE) ${ }^{49}$ has been proposed as another preparation method of SiNWs due to its low-cost fabrication process with easy controllability and reproducibility. In this technique, the Si atoms under the noble metal are oxidized due to hole injection, where a rapid etching rate of $\mathrm{Si}$ is observed in $\mathrm{HF} / \mathrm{H}_{2} \mathrm{O}_{2}$ solution. Hence, the diameter of the nanowires is dependent on the size of the noble metal particles and their length is determined by the etching solution and etching time.

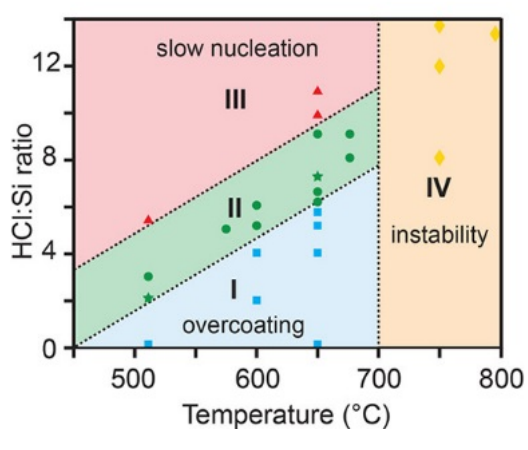

(a)
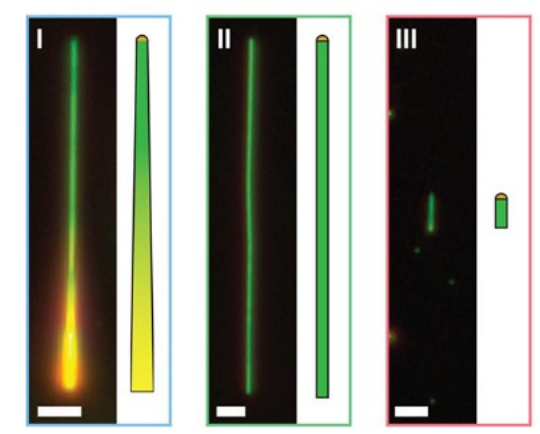

(b)
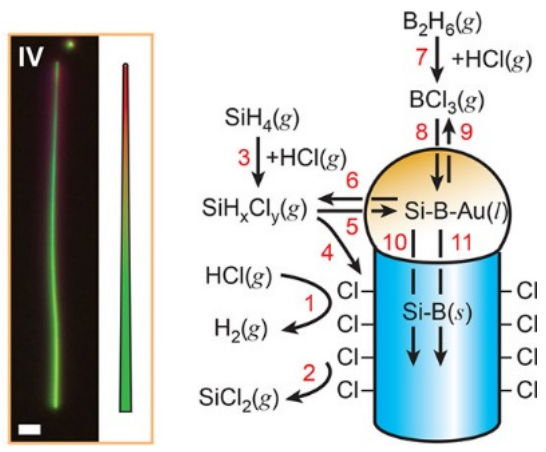

(c)

Fig. 2. (a) Qualitative analysis of $p$-type NW growth conditions depended on temperature and $\mathrm{HCl}_{\mathrm{SiH}} \mathrm{S}_{\mathrm{H}}$ ratio. (b) Dark-field microscopy images (left) and schematics (right) exemplifying NWs grown in regimes I-IV, scale bars, $4 \mu \mathrm{m}$. (c) Schematic of the microscopic chemical process that can occur during $p$-type NW growth. Reprinted with permission from Ref. 27. Copyright 2017 ACS Appl. Mater. Interfaces. 
In 2008, Zhang et al. ${ }^{50}$ investigated the influences of different Si substrates and different etching conditions on the structural characteristics of SiNWs. The nanowires obtained after etching p-Si (100) substrates are perpendicular to the silicon substrate. While, the angle between the nanowire and the $\mathrm{Si}$ substrate is about $40^{\circ}$, as the crystal orientation of the substrate with (111) direction was selected. In addition, the effects of the etching solution's $\mathrm{H}_{2} \mathrm{O}_{2}$ concentration on nanowire morphology and density are important. The higher the $\mathrm{H}_{2} \mathrm{O}_{2}$ concentration, the faster the etching reaction, leading to more $\mathrm{Si}$ being dissolved.

At present, one of the major researches on the preparation of SiNWs using the MACE method is focused on how to obtain SiNWs with small diameter and large aspect ratio. Honchbaum et al. ${ }^{51}$ prepared SiNWs with diameters ranging from 20 to $300 \mathrm{~nm}$ and aspect ratios of up to 500 by using an $\mathrm{AgNO}_{3}$ and $\mathrm{HF}$ acid mixture. However, the distribution of nanowire diameters was large and the size uniformity was poor. In 2015, ultrathin SiNW arrays were fabricated by Tan et al. ${ }^{33}$ using ion beam-assisted chemical etching. As shown in Fig. 3, the average diameters were less than $10 \mathrm{~nm}$ and the aspect ratios reached 1600. In their experiment, after implanting $\mathrm{Ag}$ ions into the thin $\mathrm{Au}$ film, which was grown on the surface of the Si substrate, large quantities of $\mathrm{Au}-\mathrm{Ag}$ particles were formed beneath the surface of the Si substrate at an appropriate dose. These $\mathrm{Au}-\mathrm{Ag}$ particles were used as catalysts in MACE for fabricating SiNWs. It can be seen from Fig. 3(c) that the diameters of SiNWs were distributed between $6 \mathrm{~nm}$ and $12 \mathrm{~nm}$, which shows that the SiNWs had a uniform distribution of diameters. And the ultrathin SiNWs could be easily gathered together due to the ultra-large aspect ratio. In this technique, ion implantation was employed to disperse $\mathrm{Au}-\mathrm{Ag}$ alloy catalysts into the Si substrate, which will greatly reduce the size of the catalysts. In addition, one-dimensional porous SiNWs were also prepared by a one-step MACE method to etch $p$-doped Si substrates. ${ }^{31}$ This nanostructure was used as the anode of a lithiumion battery, which had fast charge-discharge and superior cycling performance due to the high surface area of the anode. ${ }^{1}$

\subsection{Ordered Si nanowire arrays}

The preparation of ordered Si nanowire arrays has become a research hotspot because the photoelectric properties of nanomaterials in ordered arrangements can be improved significantly due to the collaborative effect and coupling effect. ${ }^{52}$ Additionally, nanowires in ordered arrays are separated by a certain distance rather than being gathered together. This lays a good foundation for exploring the modification of SiNWs by cladding them with another material. ${ }^{53}$

A template is necessary to prepare well-organized nanostructures in ordered arrangements. The quality of the template determines the diameter and uniformity of the ordered SiNW arrays. Currently,

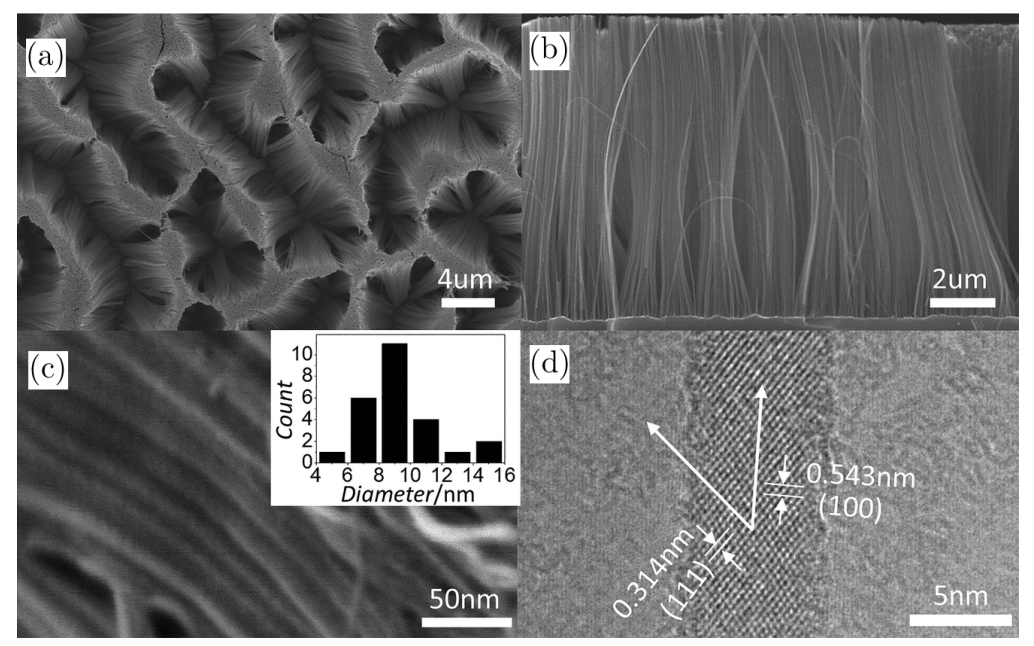

Fig. 3. (a) A top view SEM image of the ultra-thin SiNWAs fabricated using IBCE. (b) The cross-sectional SEM image of the ultrathin SiNWAs. (c) A higher magnification SEM image of the SiNWAs. The inset shows the diameter distribution of these SiNWAs. (d) A HR-TEM image of a SiNW obtained using IBCE. Reprinted with permission from Ref. 33. Copyright 2015 Nanoscale. 
common templates for the fabrication of ordered SiNW arrays mainly include anodic aluminum oxide (AAO) templates, photolithography masks, and nanosphere templates.

De et al. ${ }^{54}$ pointed out that it is easy to fabricate perfectly-ordered SiNW arrays in an area of several square centimeters by the laser interferometric lithography (LIL) technique. With more advanced equipment, a defect-free pattern can be gained on the whole $300 \mathrm{~mm}$ wafer. ${ }^{55}$ In Fig. 4 , the shape of these $\mathrm{Si}$ nanowires is appropriately square and the periodic pattern is not limited to being hexagonally symmetric, which makes them suitable for use in electronic and thermoelectric devices. In their experiment, the diameters of the Si nanowires ranged between $65 \mathrm{~nm}$ and $350 \mathrm{~nm}$ and had a surface density greater than $2 \times 10^{7} \mathrm{~mm}^{-2}$.

Park et al. proposed a bottom-up approach to fabricate ordered SiNW arrays using Au nanodiskes formed by combining $\mathrm{KrF}$ stepper lithography (KSL) and CVD followed the VLS mechanism. ${ }^{29}$

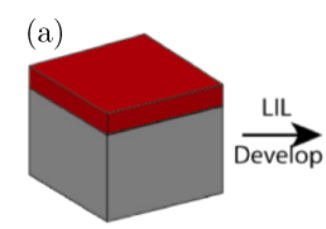

(d)
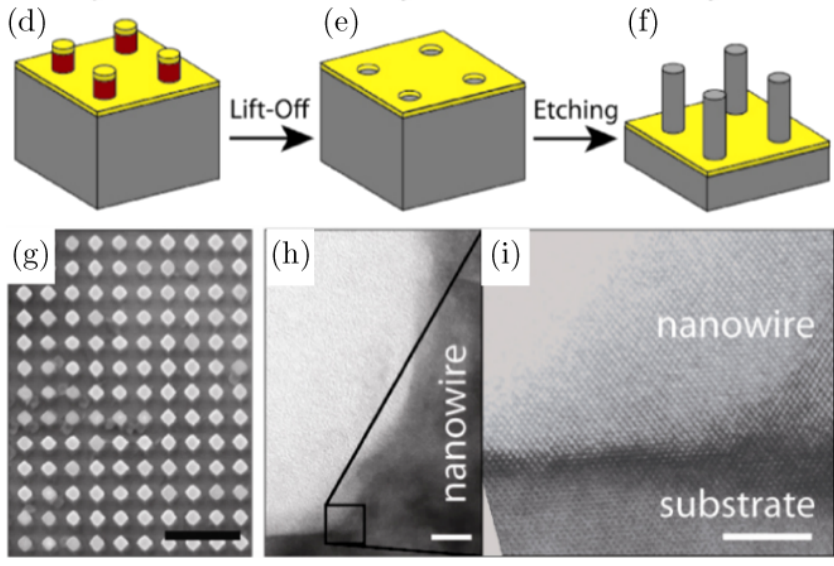

Fig. 4. (a)-(c) Laser interference lithography (LIL) combined with $\mathrm{O}_{2}$ plasma etching is employed to produce arrays of photoresist posts. (d) Metal evaporation, (e) lift-off and (f) metalassisted etching leads to vertically aligned arrays of Si nanowires, shown in $(\mathrm{g})$. The wires have a diameter of around $350 \mathrm{~nm}$, a periodicity of $710 \mathrm{~nm}$ and a length of several micrometers. The TEM image in (h) shows the foot of a nanowire and the substrate. The high-resolution magnification in (i) proves that the wire and substrate form a single crystal. The scale bars are $2 \mu \mathrm{m}, 10 \mathrm{~nm}$ and $5 \mathrm{~nm}$ in (g), (h) and (i), respectively. Reprinted with permission from Ref. 54. Copyright 2010 Nanotechnology.
The KSL technique can obtain nanometer-to-micrometer-scale patterns in an area of $1 \mathrm{~cm}^{2}$. The SiNWs arrys in a perfect hexagonal array were obtained with the diameter of $220 \mathrm{~nm}$ and length of $17 \mu \mathrm{m}$, as shown in Fig. 5. The smooth surface, straight morphology and single-crystalline of SiNWs have been proved by TEM.

Although large-area and highly-ordered SiNW arrays have already been obtained by the LIL and KSL technique, these techniques still require expensive equipment and complicated processes, which impedes the application of ordered SiNW arrays. Therefore, reducing cost becomes another expected breakthrough. Many other techniques are proposed, such as nanosphere lithography (NSL), and AAO template method.

Kim et al. ${ }^{37}$ studied the influences of an $\mathrm{Au} / \mathrm{Ag}$ bilayered metal mesh on the surface morphology of nanowires by combining the AAO template and MACE method. Often, Ag can be anodically dissolved in an etchant solution containing an HF and
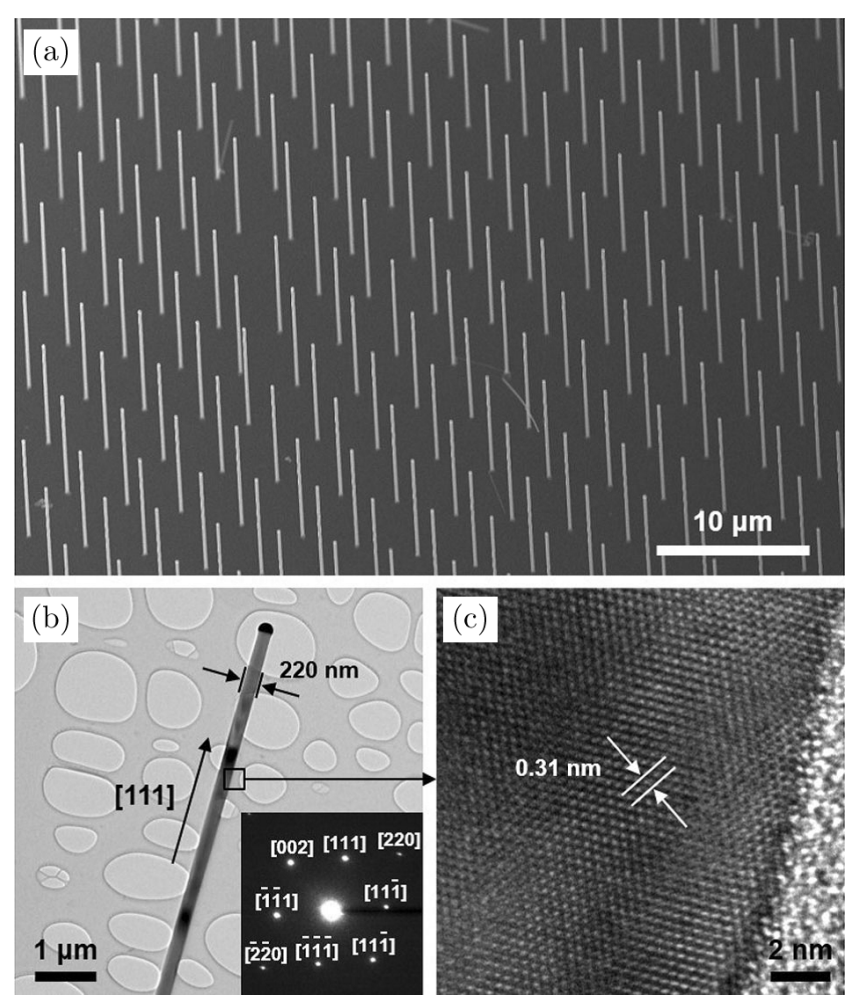

Fig. 5. (a) $20^{\circ}$ tilt-viewed SEM image of $\mathrm{v}$-SiNW arrays having high fidelity in large area. (b) Low-magnification TEM image of a SiNW. Inset of (b) shows the SAED pattern indexed for single crystalline Si. (c) HRTEM image taken from the area designated in (b). Reprinted with permission from Ref. 29. Copyright 2016 Chem. Asian J. 
$\mathrm{H}_{2} \mathrm{O}_{2}$ mixture, leading to SiNWs' surface roughening. ${ }^{50,56,57}$ To solve this problem, they proposed a novel metal mesh structure with an $\mathrm{Au} / \mathrm{Ag}$-stacking configuration. In this structure, Ag film contacts the polished surface of the Si substrate. During MACE reaction, $\mathrm{Au}$ is inert against oxidative dissolution in a solution of $\mathrm{HF}$ and $\mathrm{H}_{2} \mathrm{O}_{2}$. Thus, the upper $\mathrm{Au}$ layer effectively prevents not only undesired structural disintegration of the underlying Ag layer, but also tapering of the SiNWs. Furthermore, Au film can still act as a catalyst for $\mathrm{H}_{2} \mathrm{O}_{2}$ decomposition on an Ag surface. Figure 6 shows that the surface of the SiNWs is relatively smooth, and the sizes of the nanowires at the top and bottom are consistent without showing a tapered morphology. This is attributed to the inert nature of the Au layers in the bilayered metal mesh against anodic dissolution.

To gain smaller SiNWs in ordered arrays, Huang et al. ${ }^{18}$ prepared ordered SiNWs arrays with an average diameter of about $10 \mathrm{~nm}$ by controlling the pore size in the AAO template and the metal thickness. The distribution of these diameters ranged between $8 \mathrm{~nm}$ and $20 \mathrm{~nm}$, and their surface densities were up to $10^{10} \mathrm{~cm}^{-2}$. The surfaces of the
SiNWs are smooth, but the uniformity of nanowire heights is poor.

NSL is another potential technique for fabricating ordered SiNW arrays and has the advantages of low cost and simple operation. This approach starts with the self-assembly of a monolayer of polystyrene (PS) spheres arrayed on a Si substrate. The pattern of this array has a hexagonal close-packed structure. Fuhrmann et al. fabricated the ordered arrays of SiNW by using NSL without reducing their size and MBE followed the VLS mechanism. ${ }^{28}$ Gold was directly deposited on the hexagonal close-packed nanosphere arrays. After annealing, the nanosphere was removed and ordered arrays of gold particles was formed. With the deposition of $\mathrm{Si}$ atoms by MBE, SiNWs with diameter of $70 \mathrm{~nm}$ and length of $250 \mathrm{~nm}$ were synthesized by the catalysis of gold. The arrangement of SiNWs displays a hexagonal structure.

In addition, the size of PS spheres in the arrays can be reduced by reactive ion etching (RIE), ${ }^{34}$ plasma etching ${ }^{58}$ or ion beam etching, ${ }^{59}$ which transfers the close-packed spheres into nonclosepacked ones. In this way, the size of SiNWs and
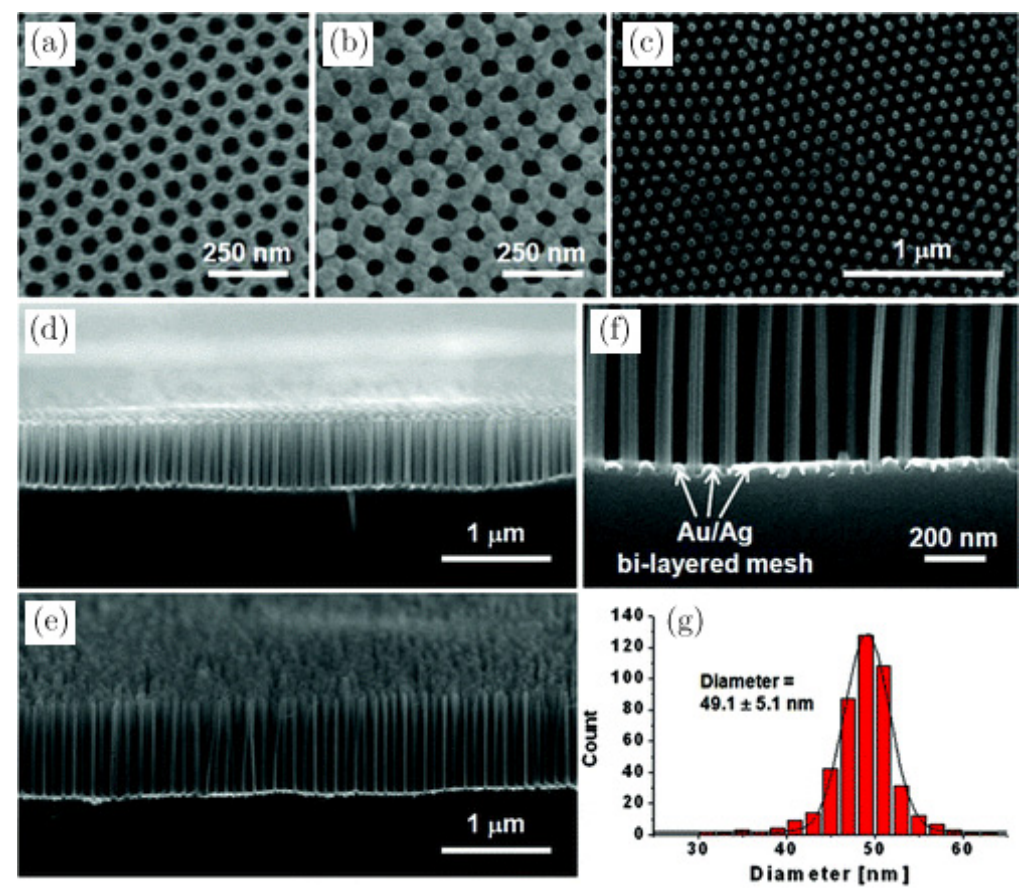

Fig. 6. SEM micrographs of (a) AAO membrane and (b) Au/Ag bilayered metal mesh. (c) Typical plan-view SEM image of extended arrays of vertically aligned SiNWs obtained by MaCE of $\mathrm{Si}(100)$ wafers by using $\mathrm{Au} / \mathrm{Ag}$ bilayered metal mesh. (d,e) Cross-sectional SEM images of vertically etched $\mathrm{Si}(100)$ wafers, showing SiNWs with different diameters; (d) $63.9 \pm 9.2$ and (e) $39.5 \pm 4.2 \mathrm{~nm}$. (f) A magnified cross-sectional SEM image of a vertically etched Si(100) wafer taken near the etching front. (g) Histogram showing the diameter distribution of SiNWs shown in panel c, together with a Gaussian fit (solid line) of the measured statistical data. Reprinted with permission from Ref. 37. Copyright 2011 Acs Nano. 
interval distance between SiNWs can be modulated according to the need of experiment. In 2007, Huang et $a l .{ }^{34}$ fabricated larger scale SiNW arrays with controlled nanowire diameters, lengths and densities. In their research, the diameter of the PS nanospheres decreased from $360 \mathrm{~nm}$ to $180 \mathrm{~nm}$ by adjusting the time of RIE. The length of nanowires increased from $1.3 \mu \mathrm{m}$ to $5.3 \mu \mathrm{m}$ with an increase of MACE etching time. However, after the diameter of the PS spheres decreased to nearly $50 \mathrm{~nm}$, the morphology of the nanospheres became irregular rather than circular, which is disadvantageous for the preparation of SiNWs with uniform shape. In order to gain small diameter nanowires, $\mathrm{Li}$ et al. ${ }^{38}$ proposed a novel method combining chemical etching and thermal oxidation. In their experiments, SiNWs with diameters of $95-200 \mathrm{~nm}$ were first prepared by the conventional NSL technique. After oxidizing, the SiNWs at $950^{\circ} \mathrm{C}$ for $1 \mathrm{~h}, \mathrm{Si} / \mathrm{SiO}_{2}$ core-shell nanowires were formed. $\mathrm{The}^{\mathrm{SiO}_{2}}$ shell on the SiNWs was removed by a buffered oxide etching solution $\left(40 \% \mathrm{NH}_{4} \mathrm{~F}: 49 \% \mathrm{HF}=6: 1\right)$ for $3 \mathrm{~min}$. As a result, vertically aligned, sub-20 nm SiNW arrays were formed. In Fig. 7(a), tapered SiNWs are evident, which may be attributed to accelerated oxidation at the nanowire tip.

Most studies on the preparation of ordered SiNW arrays are focused on the fabrication of vertical and straight nanowires. However, some ordered SiNW arrays with novel structures have been reported recently, such as kinked ${ }^{40}$ and zigzag nanowires. ${ }^{41}$ In order to form these nonstraight nanowires, an alternating MACE method was proposed by Chen et $a l .{ }^{40}$ In this approach, a Si substrate is etched alternately by different types of etchant. By adding some organic additives into the etchant, such as methyl alcohol, ethyl alcohol, 2-propanol or
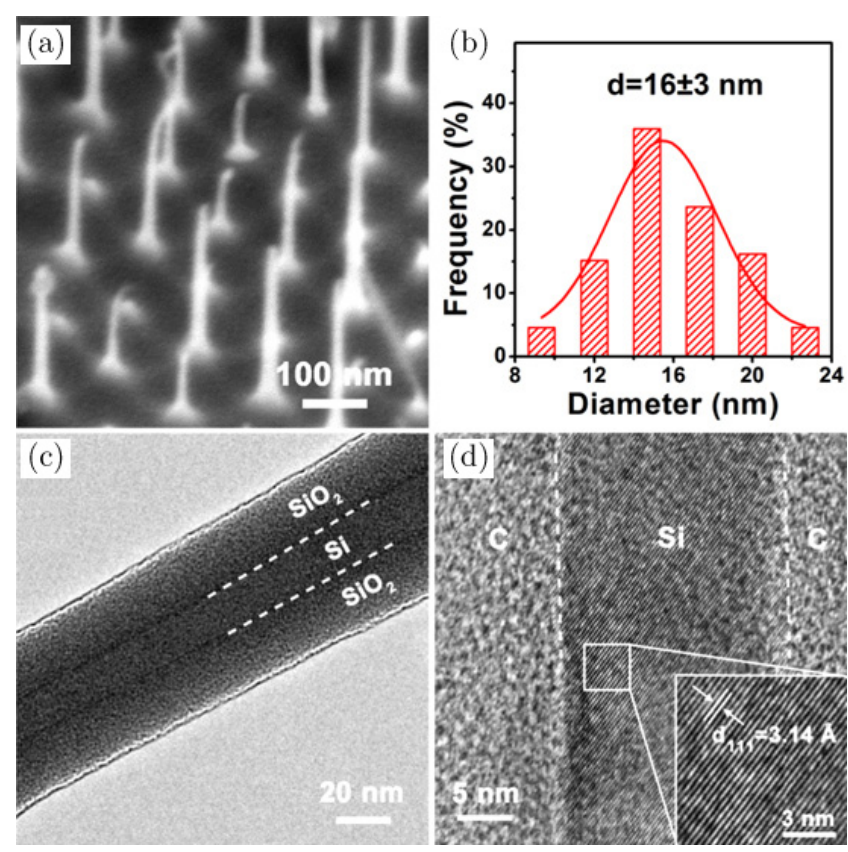

Fig. 7. (a) SEM image of sub-20 nm SiNWs fabricated after two-step oxidation (at $950^{\circ} \mathrm{C}$ for $60 \mathrm{~min}$ and $20 \mathrm{~min}$, respectively) and $\mathrm{SiO} 2$ shell removal; (b) size distribution of the sub$20 \mathrm{~nm}$ SiNWs. More than $150 \mathrm{NWs}$ were counted to obtain adequate statistics; (c) TEM image of a single sub-20 nm SiNW without removing the $\mathrm{SiO} 2$ shell; (d) HRTEM image of a single sub-20 nm SiNW on supporting TEM carbon film after SiO2 shell removal. The inset is a high-magnification HRTEM image showing lattice fringes. Reprinted with permission from Ref. 38. Copyright 2016 Nanotechnology.

acetonitrile, the etching direction will be changed because organic additives can lower the surface tension of the etchants and decrease the etching rate along the vertical direction. Kinked nanowires with two or more kinks, with segments of the same or different lengths, and with small or large kinking angles, are shown in Fig. 8(a). The number of kinking points is determined by the number of times

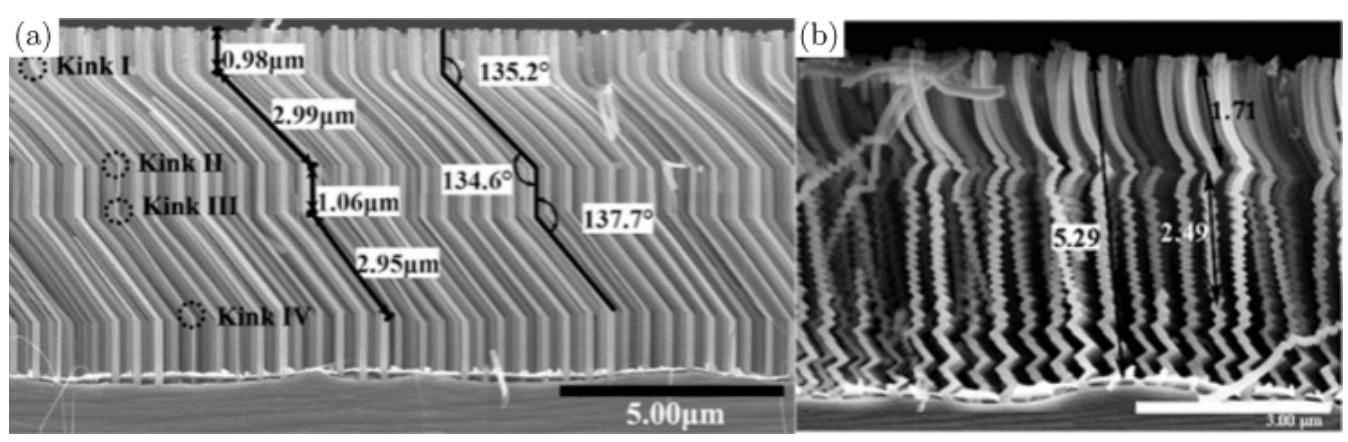

Fig. 8. (a) Periodically multiply kinked nanowires with four kinks after changing etchant four times; (b) Zigzag NWs formed in $10 \mathrm{~mL}$ of $\mathrm{HF}, 2 \mathrm{~mL}$ of $\mathrm{H}_{2} \mathrm{O}_{2}, 5 \mathrm{~mL}$ of glycerol and $15 \mathrm{~mL}$ of DI water with etching time is 10 min. Reprinted with permission from Refs. 40 and 41. Copyright 2017 Nano Letters and 2017 Nano Letters. 
the type of etchant is changed. The angle of the kinks is related to the surface tension and viscosity of the etchant. Moreover, they also developed a diffusioncontrolled MACE method to fabricate another form of nonstraight nanowires - zigzag nanowires, as shown in Fig. 8(b). By adjusting the composition of etchant to change its diffusivity, etching direction and etching time, various zigzag nanowires were formed. ${ }^{41}$ They found that the etchant's viscosity plays a significant role in zigzag nanowire fabrication.

\section{Preparation and Structures of GeQDs on Planar Substrate}

Since the dislocation-free GeQDs were proven by Eaglesham and Mo et al. ${ }^{60,61}$ via TEM in 1990, GeQDs have attracted wide attention because of their special properties, such as their small band gap, nontoxicity, compatibility with well-developed Si technology and the long service life of their carriers. ${ }^{62,63}$ Over the last three decades, a deep understanding of Ge growth mode, the evolution of island size, density, shape, and the relationship between components and strain under various conditions has been attained. ${ }^{60-82}$ The growth mechanisms of Ge islands have been analyzed using thermodynamic and kinetic theories. ${ }^{65-67}$ Furthermore, by modulating their optical and electrical properties, many photoelectric and electronic devices, such as infrared detectors, ${ }^{68}$ near-infrared light emitting diodes ${ }^{69}$ and single-electron transistors ${ }^{70}$ have been explored.

Some research progress on the microstructural and optical properties of Ge quantum dots over the past five years is listed in Table 2. Clearly, current researches mainly focus on the improvement of

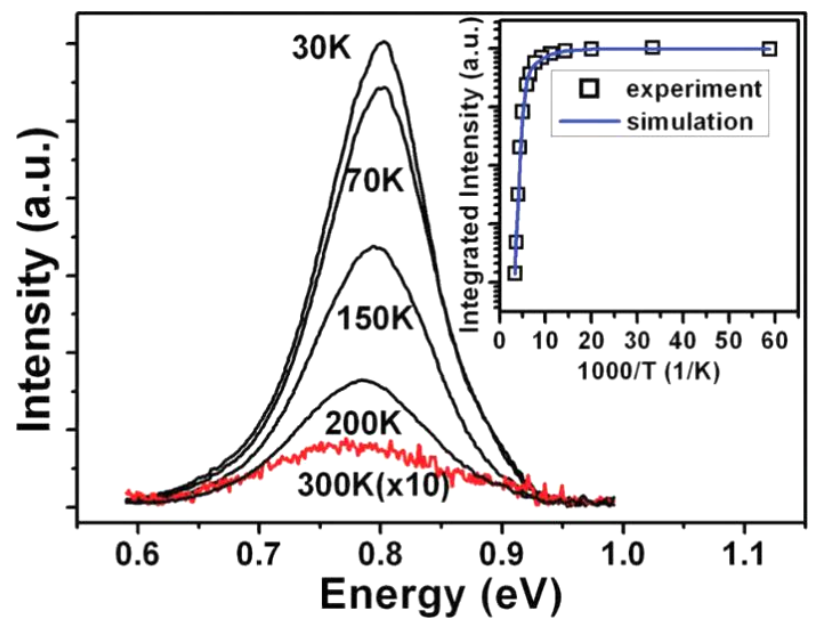

Fig. 9. PL spectra of GeSi QDs with $0.9 \mathrm{~nm}$ Ge deposition on a miscut $\mathrm{Si}(001) / 4^{\circ}$ substrate at 30, 70150,200 and $300 \mathrm{~K}$. The spectrum at $300 \mathrm{~K}$ is multiplied by a factor of 10 . The integrated intensity of PL peak versus the temperature is shown in the inset, which is fit by $I(T)=I(0) /\left[1+C_{1}\left(\exp \left(-E_{1} / k T\right)+\right.\right.$ $C_{2}\left(\exp \left(-E_{2} / k T\right)\right]$. Reprinted with permission from Ref. 71. Copyright 2014 APL Materials.

luminescence properties by acquiring quantum dots with high density, uniform sizes and other special structures. Zhou et al. ${ }^{71}$ observed a quite strong Photoluminescence (PL) peak of dense GeQDs on a miss-cut substrate even at $300 \mathrm{~K}$, as shown in Fig. 9, which was attributed to the good confinement of excitons in dense GeQDs due to the absence of the wetting layer. The absence of the wetting layer also leads to a transition of the growth mode from the Stranski-Krastanov (SK) mode to the VolmerWeber (VW) mode. Extremely dense QDs with a density of $2 \times 10^{11} \mathrm{~cm}^{-2}$ were formed on the misscut $\mathrm{Si}$ substrate with $6^{\circ}$. The steps induced by the miss-orientation of the Si substrate can significantly

Table 2. Statistical results of some research results on the microstructure and optical properties of Ge quantum dots in the past years.

\begin{tabular}{|c|c|c|c|c|c|c|c|}
\hline Structure characteristic & $\begin{array}{c}\text { Layer } \\
\text { number }\end{array}$ & Year & Density $\left(\mathrm{cm}^{-2}\right)$ & Size $(\mathrm{nm})$ & PL temperature & $\begin{array}{c}\text { PL intensity } \\
\text { enhanced }\end{array}$ & Ref. \\
\hline QDs on miscut substrate & Single & 2014 & $1 \times 10^{11}$ & / & RT & $\mathrm{Y}$ & 71 \\
\hline Circularly ordered array & Single & 2011 & $5 \times 10^{11}$ & $40-75$ & $30-200 \mathrm{~K}$ & $\mathrm{Y}$ & 72 \\
\hline Ultrasmall size & Single & 2012 & / & 8 & $15-100 \mathrm{~K}$ & / & 73 \\
\hline QDs on RSG virtual substrate & Single & 2014 & / & / & $175 \mathrm{~K}$ & / & 74 \\
\hline Super-dense array & Single & 2014 & $8 \times 10^{12}$ & 3 & / & / & 76 \\
\hline QDs+B ions implantation & Single & 2014 & / & / & / & $\mathrm{Y}$ & 76 \\
\hline $\mathrm{Ge} / \mathrm{Si} / \mathrm{Ge} \mathrm{CQDs}$ & Multi & 2015 & / & 24 & / & $\mathrm{Y}$ & 77 \\
\hline 3D QD crystals & Multi & 2015 & $8 \times 10^{10}$ & / & / & $\mathrm{Y}$ & 78 \\
\hline QDs+Ge ion bombarded & Multi & 2017 & / & / & $\mathrm{RT}$ & $\mathrm{Y}$ & 79 \\
\hline Disk + small laterally ordered & Multi & 2017 & / & $50-140$ & $\mathrm{RT}$ & / & 80 \\
\hline
\end{tabular}


reduce the surface diffusion length of adatoms and favor the formation of dense GeQDs.

In 2015, Kuo et $a .^{77}$ designed a novel structure with multifold $\mathrm{Ge} / \mathrm{Si} / \mathrm{Ge}$ composite quantum dot (CQD)-stacked heterostructures, which was able to effectively suppress dislocation generation in superlattice materials. The structure of multifold CQD is shown in Fig. 10. Multifold CQD refer to the overlapped growth of $\mathrm{Ge}$ and Si layers in a single quantum dot. Unlike traditional Ge quantum dots, a Si spacer is inserted into two Ge layers in a doublefold CQD. The interface between the $\mathrm{Ge}$ and $\mathrm{Si}$ layers is kept clear by controlling the growth parameters. Similarly, three layers of Ge thin film are separated by two layers of $\mathrm{Si}$ spacer in a triplefold CQD. These multifold Ge/Si/Ge CQD were employed to grow 40-period multilayered quantum dots with a 50-nm thick buffer layer. PL spectra show that increases in the fold number per stack in the CQD-stacked heterostructures significantly improve the $\mathrm{PL}$ intensity with invisible $\mathrm{PL}$ broadening, whereas the TO/NP peaks from the $\mathrm{Si}$ spacer layer become weaker. Based on these CQD, a visible-to-near-infrared photodetector for optical interconnect applications was produced. Typical responsivity values of this photodetector at $850 \mathrm{~nm}$ and $980 \mathrm{~nm}$ are $250 \mathrm{~mA} / \mathrm{W}$ and $204 \mathrm{~mA} / \mathrm{W}$, respectively. The responsivity of the $\mathrm{CQD} / \mathrm{Si}$ photodetector at $980 \mathrm{~nm}$ is greater than that of commercially-available silicon photodetectors. Improvements in photocurrent, photoresponsivity and external quantum efficiency are attributed to increased Ge volume, which facilitates efficient absorption and enhances the built-in $E$-field resulting from photocarrier confinement within the CQDs/Si system.

In 2017, Groiss et al. ${ }^{79}$ observed that the PL intensity could be improved as epitaxial GeQDs were bombarded with Ge ions in vertically stacked samples. In their design, one to two Ge ions impinged on a single quantum dot, creating an amorphic region with a depth of a few nanometers. During growth

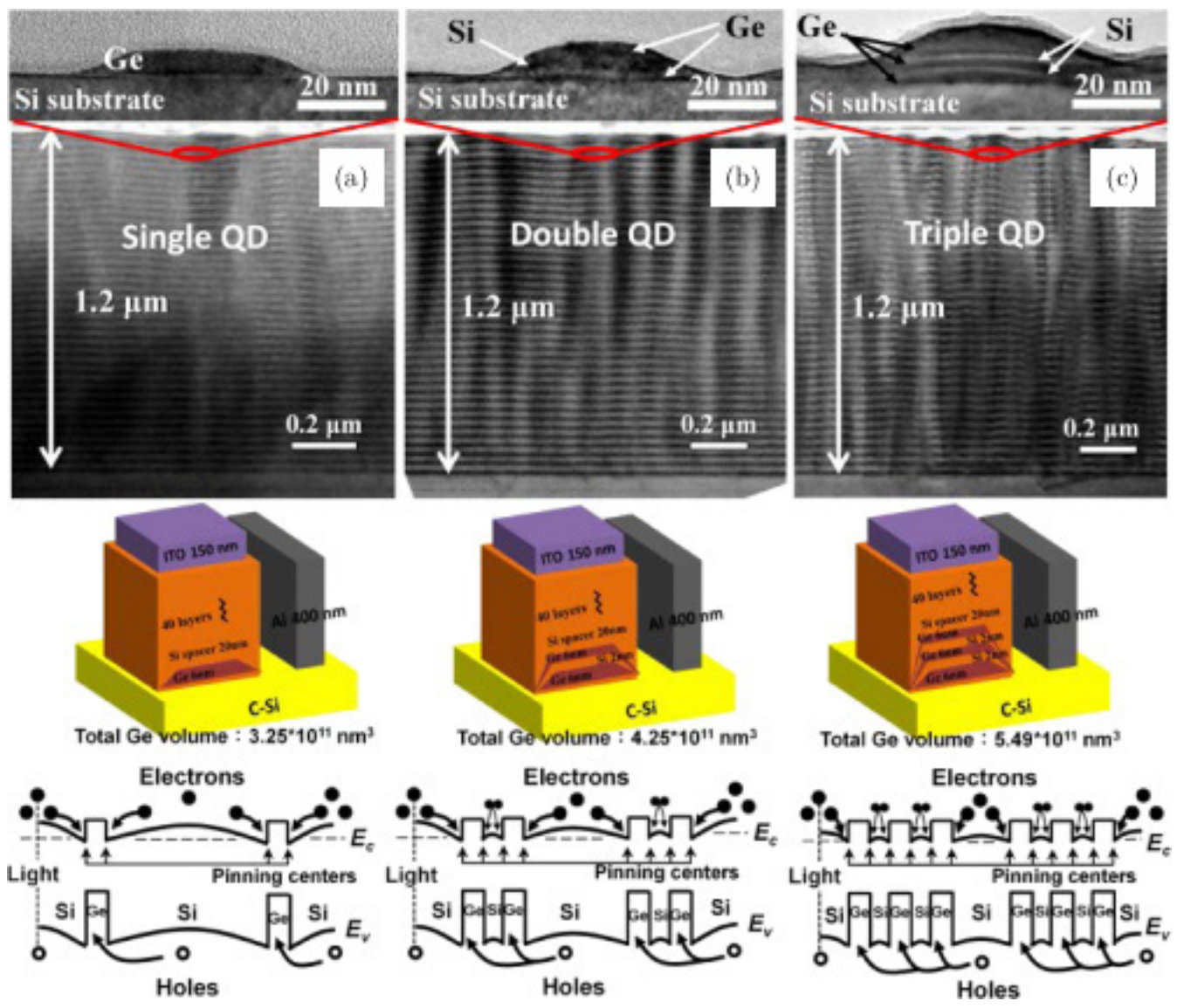

Fig. 10. CTEM micrographs, schematic structural diagrams and energy band diagrams of triple-, double-, and single-fold Ge/Si CQD heterostructures. Reprinted with permission from Ref. 77. Copyright 2015 Optical Letters. 
process, these amorphous regions partly recrystallized by solid phase epitaxial regrowth (SPER) starting from neighboring QD regions that remained crystalline. Based on the design of Ge ionbombarded epitaxial Ge quantum dots (GIB-QDs), 11-fold multi-stack samples were prepared to study the influence of the growth temperature of the $\mathrm{Si}$ spacer layers on the PL properties. As shown in Fig. 11, for samples grown at $350-500^{\circ} \mathrm{C}$, no $\mathrm{PL}$ signal is present. It shows that SPER breaks down as the temperatures are too low. The carriers are depleted by the abundance of nonradiative recombination centers resulting from dislocation. However, a strong PL peak can be observed at room temperature at the temperatures of $500-600^{\circ} \mathrm{C}$. The PL intensity for an 11-layer GIB-QD stack is $650 \%$ higher than that for single-layer samples. When the temperature is higher than $625^{\circ} \mathrm{C}$, the extended point defects can migrate out of the GIB-QD, leading to PL quenching.

In addition, the growth of GeQDs on concave substrate surface and/or convex substrate surface has also been studied with theory and experiment,${ }^{83-85}$ which lays a good foundation for exploring the growth of GeQDs on the sidewall of SiNWs. Li et al. ${ }^{84}$ developed a theoretical model to elucidate GeQDs formation on different curved patterned substrate surface. They pointed out that

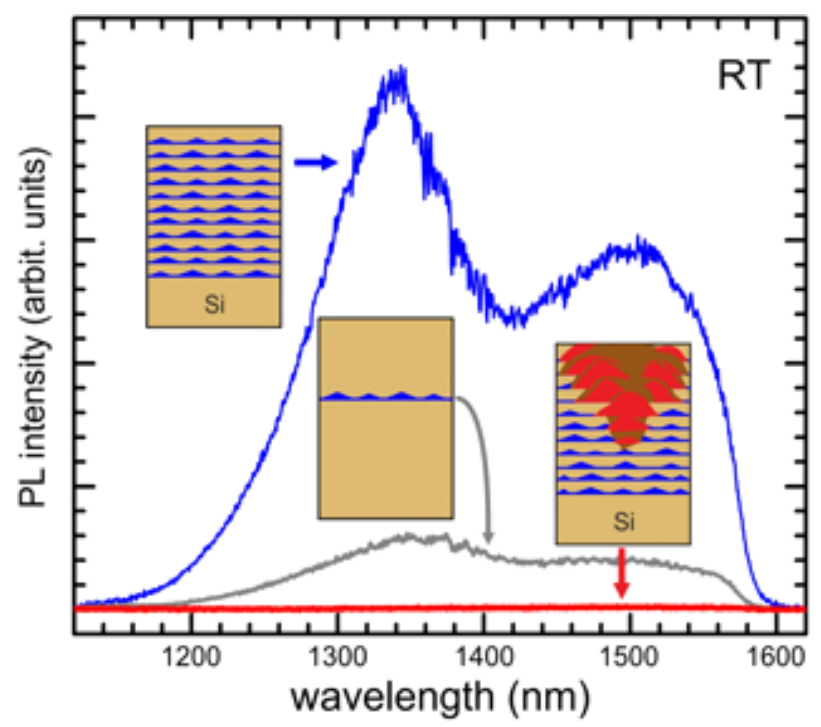

Fig. 11. Room-temperature PL spectra from a single layer of GIBQDs (gray) and from a 11-fold multi-stack (blue) for which TSi was $500-600^{\circ} \mathrm{C}$. For the multi-stack sample for which the growth parameters were not optimized $\left(\mathrm{TSi}=350-500^{\circ} \mathrm{C}\right)$, no PL signal was recorded (red) (color online). Reprinted with permission from Ref. 79. Copyright 2017 Semiconductor Science Technology. the formation energy of GeQD on a convex substrate was lower than that on a planar and concave substrate surface, as shown in Fig. 12, when the occupancy area of GeQD was larger than the periodicity of the patterned substrate surface. It suggested that the formation of GeQD on a convex substrate surface was more favorable. Conversely, when the occupancy area was smaller than the periodicity of the patterned surface, the formation

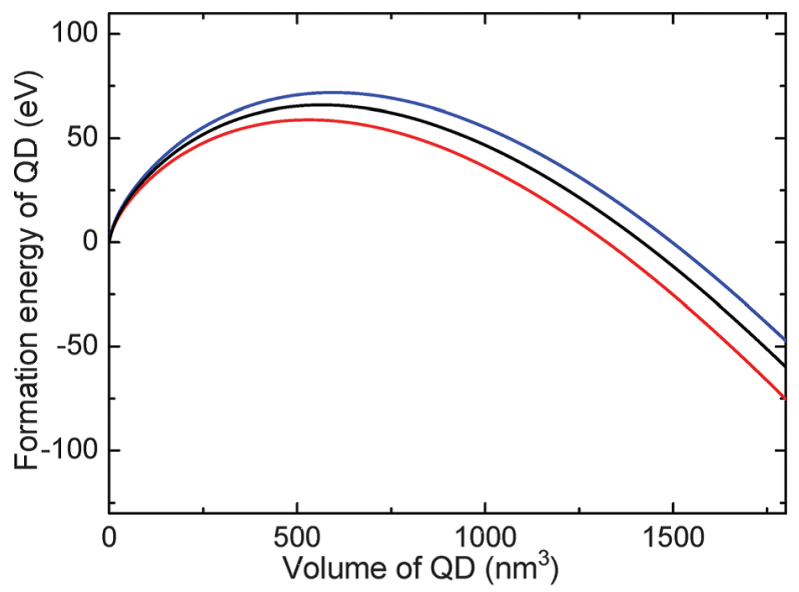

(a)

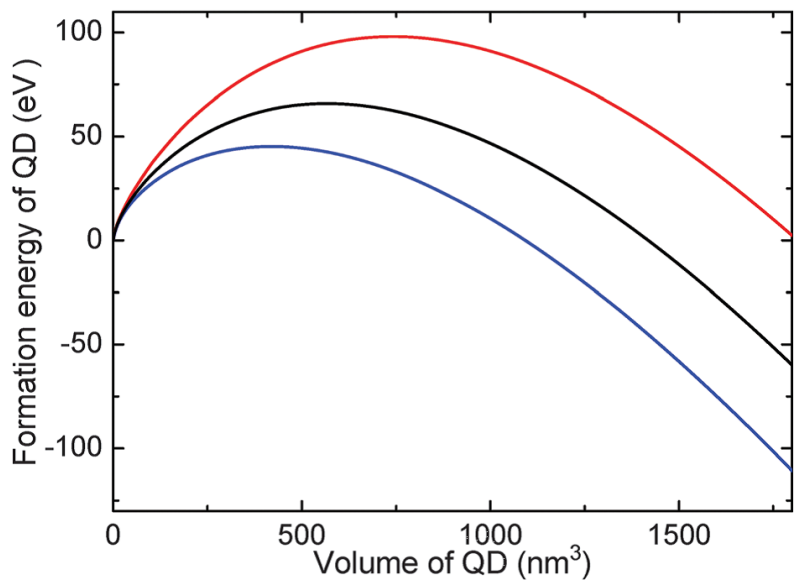

(b)

Fig. 12. QD formation energy as a function of $\mathrm{QD}$ volume on three different substrate surface curvature, $-0.01 \mathrm{~nm}^{-1}$ (blue line), 0 (black line), and $0.01 \mathrm{~nm}^{-1}$ (red line). (a) Occupancy area by $\mathrm{QD}$ is larger than the periodicity of the patterned substrate surface; (b) occupancy area by QD is much smaller than the periodicity of the patterned substrate surface. In our calculation, the shape of QD is pyramid and contact angle $\alpha=11.3^{\circ}$. According to the geometrical relations, shape factors, $A_{1}, A_{2}$ and $A_{3}$, can be given by $A_{2}=(6 / \tan \alpha)^{2 / 3}$, $A_{1}=A_{2} / \cos \alpha, A_{3}=0.9922 \tan \alpha$ (color online). Reprinted with permission from Ref. 84. Copyright 2013 Physical Chemistry Chemical Physics. 
energy of GeQD on a concave substrate surface is lower than that on a planar and convex substrate surface, which suggested that it was more favorable for GeQD to form on a concave substrate surface.

\section{SiNW/GeQD Composite Materials}

As mentioned above, as typical one-dimensional and zero-dimensional nanomaterials, SiNWs and GeQDs have already been widely studied, which lays a solid foundation for exploring the preparation and structures of SiNW/GeQD composite materials.

In 2000, single-crystal $p$-type and $n$-type SiNWs were prepared by doping boron and phosphorus elements in nanowires. ${ }^{86}$ Base on this, axial $p$ - $n$ junction nanowire photodetectors were reported in 2011 by Kim et al. ${ }^{87}$ which invoked research interest in SiNW photoelectronic devices. Furthermore, because $p$ - $i$ - $n$ junction devices have higher detection sensitivity and luminous efficiency than $p$ - $n$ junction devices, as a desirable intrinsic material, GeQDs have great potential to be integrated into SiNW devices. In this chapter, the growth mechanism and structures of SiNW/GeQD composite materials will be discussed from two aspects: QD growth on (1) radial and (2) axial surface of SiNWs.

\subsection{Coaxial SiNW/GeQD composite materials}

\subsubsection{Theoretical studies}

It is well known that the growth of a Ge thin film on a flat silicon substrate follows the S-K mode due to the $4.2 \%$ lattice mismatch between $\mathrm{Ge}$ and $\mathrm{Si}$. Growth starts in a layer-by-layer mode up to a critical thickness of about $0.4-0.6 \mathrm{~nm}$. Then, the strain energy that accumulates with an increasing thickness of $\mathrm{Ge}$ is partially relaxed through the formation of GeQDs. ${ }^{13,14}$ Nevertheless, the radial surfaces of SiNWs are not flat and have a certain nanoscale curvature. Existing theoretical models of the growth of Ge layers on planar and patterned substrates are not appropriate for such growth on surfaces with nanoscale curvatures. Therefore, new theoretical models are urgently needed to understand the basic physics involved in strain-induced growth on the radial surfaces of SiNWs.

In 2012, Fukata et al. ${ }^{12}$ calculated the stresses in the $\mathrm{Si}$ core and $\mathrm{Ge}$ shell regions of $\mathrm{Si} / \mathrm{Ge}$ core-shell nanowires by analyzing X-ray diffraction measurements. Figure 13 shows the evolution of the average lattice constants of $\mathrm{Si}$ cores and Ge shells with increasing shell growth time. It can be seen from Fig. 13(a) that the average lattice constant of $\mathrm{Si}$ cores is greater than that of bulk silicon and increases with shell growth time. This indicates that Si cores bear tensile stresses, as the Ge shells grow on the radial surfaces of nanowires. The tensile stresses increase with Ge shell thickness. In Fig. 13(b), the average lattice constant of Ge shells is lower than that of bulk Ge and increases with shell growth time. The Ge shell bears compressive stresses originating in the $\mathrm{Si}$ core. The compressive stresses are weakened by increasing the Ge shell thickness. These results indicate that the lattice mismatch between Si cores and Ge shells may be less than $4.2 \%$. Consequently, the critical thickness of

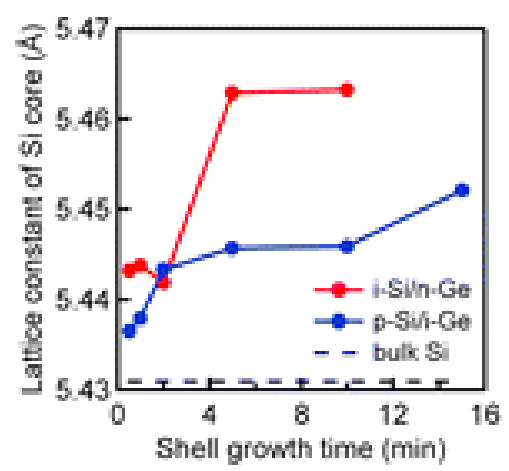

(a)

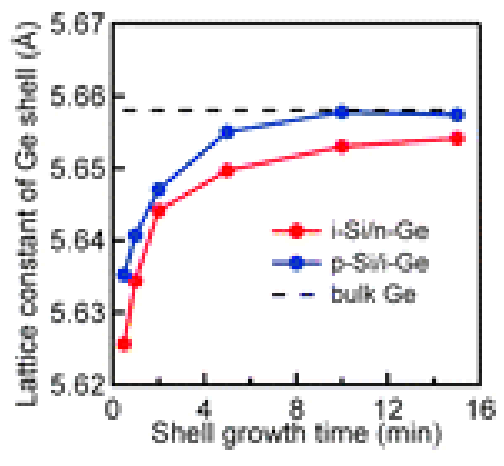

(b)

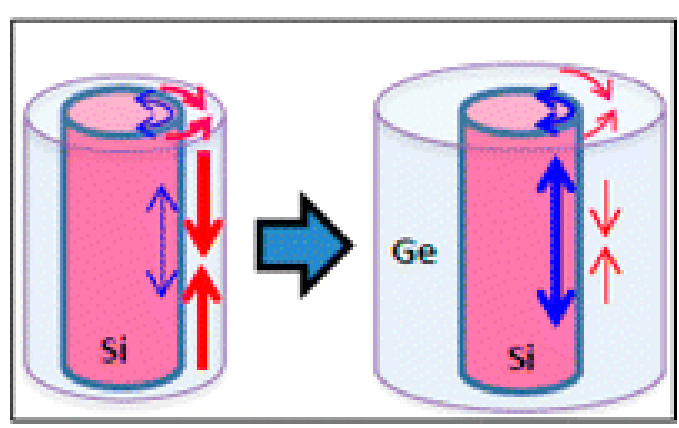

(c)

Fig. 13. Dependence of the average lattice constant of $\mathrm{Si} / \mathrm{Ge}$ core_shell NWs on the shell growth time. The lattice constant of (a) the Si core in the i-Si/n-Ge and p-Si/i-Ge core_shell NWs, (b) the Ge shell in the i-Si/n-Ge and p-Si/i-Ge core_shell NWs. (c) Schematic illustration of the stress induced by the formation of Si/Ge core-shell NWs. Reprinted with permission from Ref. 12. Copyright 2012 ACS Nano. 
the Ge shell for S-K growth is higher than that of Ge thin film grown on a planar surface. This implies that heteroepitaxial growth on the radial surfaces of SiNWs can accommodate larger stress.

The smaller the nanowire diameter, the larger the stress effects. Thus, the modified growth of the Ge shell strongly depends on the diameter of the nanowires. In 2014, Li et al. ${ }^{13}$ established a theoretical model to interpret strain-induced growth on the radial surfaces of SiNWs with increasing nanowire diameters. In their calculations, a twodimensional layer with a thickness of $\theta$ monolayers was considered on a curved surface. The energy required per atom to add a layer from the islands at a chemical potential of $\mu_{\text {island }}$ is

$$
\Delta \mu=\mu_{\text {layer }}-\mu_{\text {island }},
$$

where $\mu_{\text {layer }}$ is the energy change of the epitaxial layer from $n$ atoms to $(n+1)$ atoms. To minimize the total energy and achieve a stable condition, the system needs to balance the energies of the layer and islands. Therefore, the condition for stability is

$$
\mu_{\text {layer }}=\mu_{\text {island }} \text {. }
$$

If $\mu_{\text {layer }}<\mu_{\text {island }}$, the layer is going to grow thicker, while if $\mu_{\text {layer }}>\mu_{\text {island }}$, the layer will not grow and island growth is favored. The chemical potential of the bulk epitaxial material with no strain is used as the reference value $(\mu=0)$ in order to determine the values of $\mu_{\text {layer }}$ and $\mu_{\text {island }}$. In this case, the energy changes of an epitaxial layer from $n$ atoms to $(n+1)$ atoms is determined by the surface energy and elastic strain energy of the layer:

$$
\mu_{\text {layer }}=\Omega \gamma(h) k+\Omega \frac{\partial \gamma(h)}{\partial h}+\Omega \varepsilon_{\text {layer }},
$$

where $\Omega$ is the atomic volume, $\gamma(h)$ is the surface energy density of the layer with thickness $h\left(h=\theta h_{0}\right.$ and $h_{0}$ is the thickness of the monolayer (ML), ${ }^{88} k$ is the surface curvature and $\varepsilon_{\text {layer }}$ is the elastic strain energy density. The strain energy density can be calculated as $\epsilon_{\text {layer }}=2 G[(1+v) /(1-v)] \varepsilon^{2},{ }^{84}$ where $G$ and $v$ are the shear modulus and Poisson's ratio, and $\varepsilon$ is the strain mismatch of the layer. The first term reflects the influences of surface curvature and the second term represents changes in surface energy with layer thickness. The last term represents the contribution of the elastic strain energy.

Based on the above physical model, they studied the relationship between the growth mode of $\mathrm{Ge}$ shell layer on the radial surfaces and the nanowire

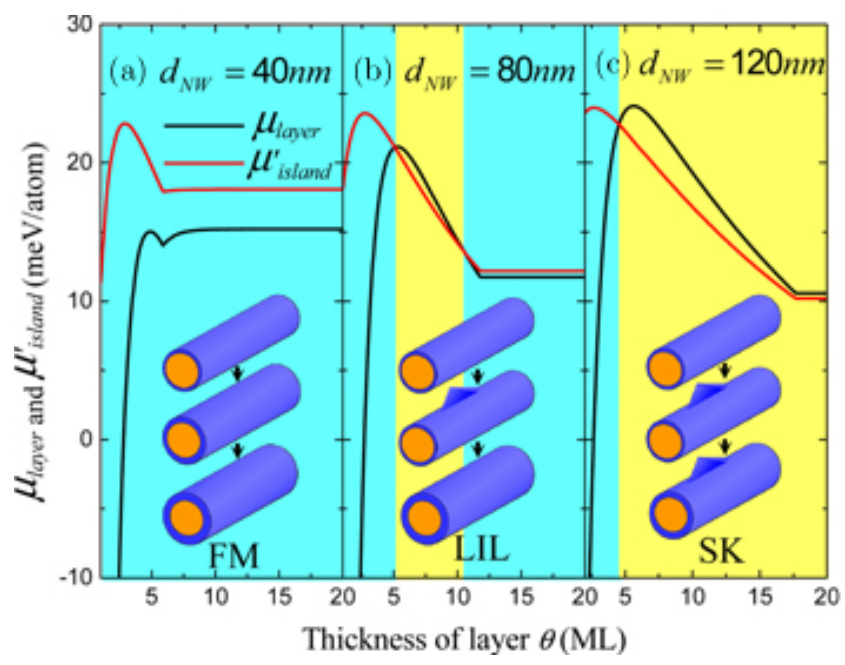

Fig. 14. The values of $\mu$ layer and $\mu^{\prime}$ island as the function of the Ge layer thickness on the surface of Si NWs with different diameters of (a) $40 \mathrm{~nm}$, (b) $80 \mathrm{~nm}$ and (c) $120 \mathrm{~nm}$. The insets in (a), (b) and (c) show the schematic three growth modes, the FM mode, the LIL mode and the SK mode, respectively. Reprinted with permission from Ref. 13. Copyright 2014 Nanotechnology.

diameter. Figure 14 displays the values of $\mu_{\text {layer }}$ and $\mu_{\text {island }}^{\prime}$ as a function of the Ge thickness with various diameters. The chemical potential of a layer is always smaller than that of an island at a diameter of $40 \mathrm{~nm}$. Generally, the growth mode of a Ge thin film is inclined to be one with lower chemical potential. Thus, it is unfavorable to form islands on such thin nanowires. At this moment, the Frank-van der Merwe (F-M) growth mode of Ge film on the radial surfaces of SiNWs is favorable. The Ge shell layer still maintains layer-by-layer growth when the thickness of Ge shell reaches $20 \mathrm{~nm}$. This is different from the growth mode of Ge film on a planar substrate. However, when the SiNW diameter is $120 \mathrm{~nm}$, the growth mode of Ge shells turns to the $\mathrm{S}-\mathrm{K}$ mode and the critical thickness for the formation of islands on the nanowire is 4 MLs. Differently, the chemical potential curves of Ge film and islands have two intersecting points for the diameter of $80 \mathrm{~nm}$ [Fig. 14(b)], indicating that a complicated transition occurs. The growth mode of Ge film transitions from "layer growth" to "island growth" and then to "layer growth" again, so this mode is named layer-island-layer (LIL). Furthermore, it is estimated from their calculations that the Ge shell layer exhibits an F-M growth mode when the diameter of the SiNWs is less than $70 \mathrm{~nm}$ but turns to the S-K mode with the diameter exceeding $100 \mathrm{~nm}$. The nanoscale curved surface of the nanowires can 
help release mismatch strain in a process of selfrelaxation. Hence, as Ge thin film grows on the radial surfaces of SiNWs, the types of mismatch strain relaxation may include Ge island relaxation, selfrelaxation of the epitaxial layer and relaxation of dislocation. The competitions among them determine the growth of Ge shells.

Additionally, the distribution of Ge quantum dots on the radial surfaces of nanowires has also been studied. Schmidt and Wang et al. ${ }^{89,90}$ used a surface perturbation model to describe the distribution pattern of GeQDs after depositing Ge thin film on a SiNW backbone. Their calculations show that at certain diameters, the misfit strain in coreshell nanowires can propagate along their length and self-assemble into a periodic pattern that minimizes the total strain energy. ${ }^{91,92}$ As shown at the top of Fig. 15, periodic GeQD arrays in one of

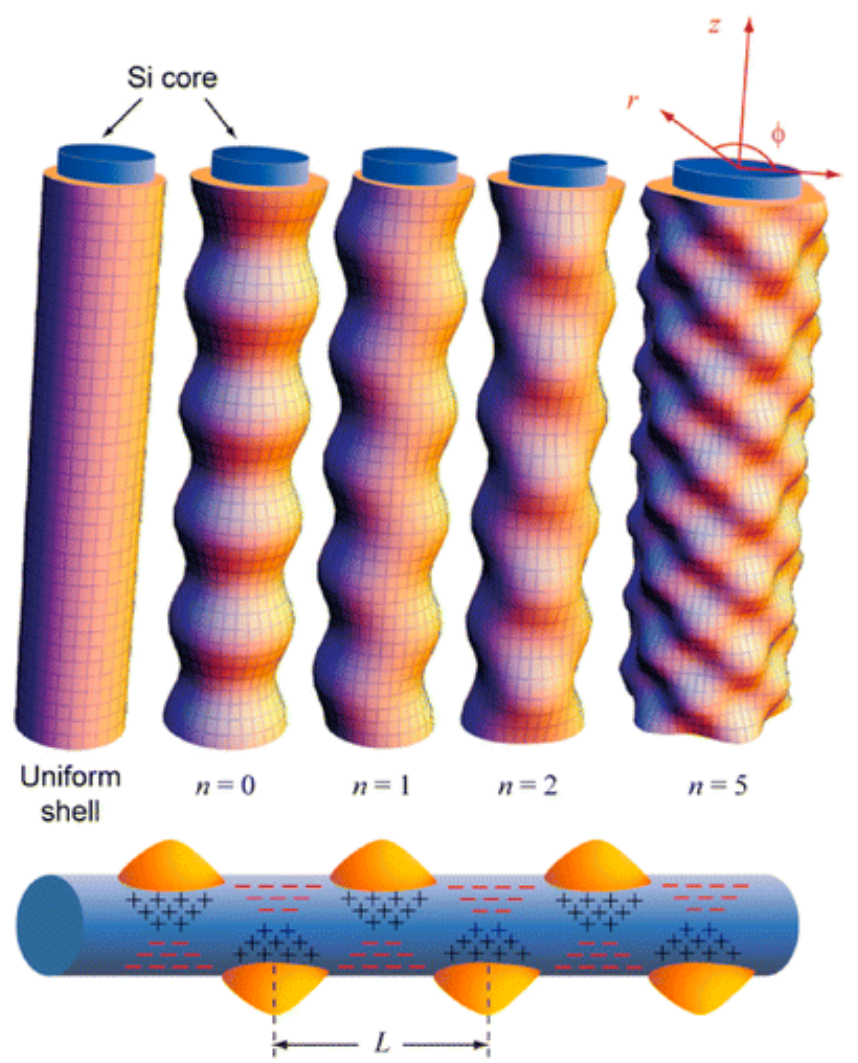

Fig. 15. (Top) Schematic of possible surface instability modes of Ge shell on Si core nanowires. Homogeneous Ge shell and four different types of inhomogeneous growth modes are shown: $n=0,1,2$ and 5. (Bottom) Alternating coherent strain distribution inside the core is shown as + (tensile) and - (compressive) signs for an $n=1$ mode growth. The QD wavelength $\mathrm{L}$ is defined as the periodic distance between QDs on the same side of the wire. Reprinted with permission from Ref. 13. Copyright 2014 Nanotechnology. several unique growth modes can be formed due to the initial instability of Ge shell layers. The nanowire diameter determines the distribution of the quantum dots. The periodic strain fields distributed into the core nanowire is corresponding to the distribution of periodic QDs arrays. For example, the alternating coherent strain distributed inside the core is shown at the bottom of Fig. 15. Because the lattice constant of $\mathrm{Ge}$ is larger than that of $\mathrm{Si}$, tensile strain ( + signs) is created in Si cores below the interfaces of GeQDs, while compressive strain (- signs) is produced, accordingly, at the opposite side. GeQDs are preferentially formed at the tensilestrained surfaces of Si cores. As a result, an anticorrelated zigzag organization of GeQD arrays is distributed on SiNWs through strain modulation, which reduces the total elastic energy of the system. Besides, the periodic distance $(\mathrm{L})$ between quantum dots at the same side is inversely proportional to the diameter of the nanowires.

\subsubsection{Experimental studies}

The fabrication of a coaxial SiNW/GeQD composite material was first reported by Pan et al. ${ }^{2}$ in 2005. In their experiment, GeQDs were grown on the radial surfaces of SiNWs using the CVD and VLS method. The growth modes of Ge thin films were proven by transmission electron microscopy (TEM). Their experimental results demonstrated that the growth mode of Ge shell layer on the sidewalls of SiNWs was determined by the diameter of the nanowires. The growth of Ge shell exhibited the F-M growth mode when the nanowire diameters ranged between $10 \mathrm{~nm}$ and $50 \mathrm{~nm}$, and formed $\mathrm{Si} / \mathrm{Ge}$ core-shell nanowire structures. However, when the diameters exceeded $50 \mathrm{~nm}$, the Ge shell showed an $\mathrm{S}-\mathrm{K}$ growth mode and formed a SiNW/GeQD composite structure. This tendency agrees with the simulation results reported by Li et al., but the LIL growth mode was not observed in the experiment. The morphology of GeQDs grown on the radial surfaces of SiNWs with a diameter of $100 \mathrm{~nm}$ is shown in Fig. 16. The shape of the GeQDs on the nanowires is asymmetric and their crystal facets are (002), (111) and (112). These facets are different from the facets of quantum dots on the planar surface, which are surrounded by (15 323$),(113)$ and (105) facets. Furthermore, several (111) stacking faults and $\{111\}$ type twins are present in the GeQDs. These stacking faults are likely bounded by 

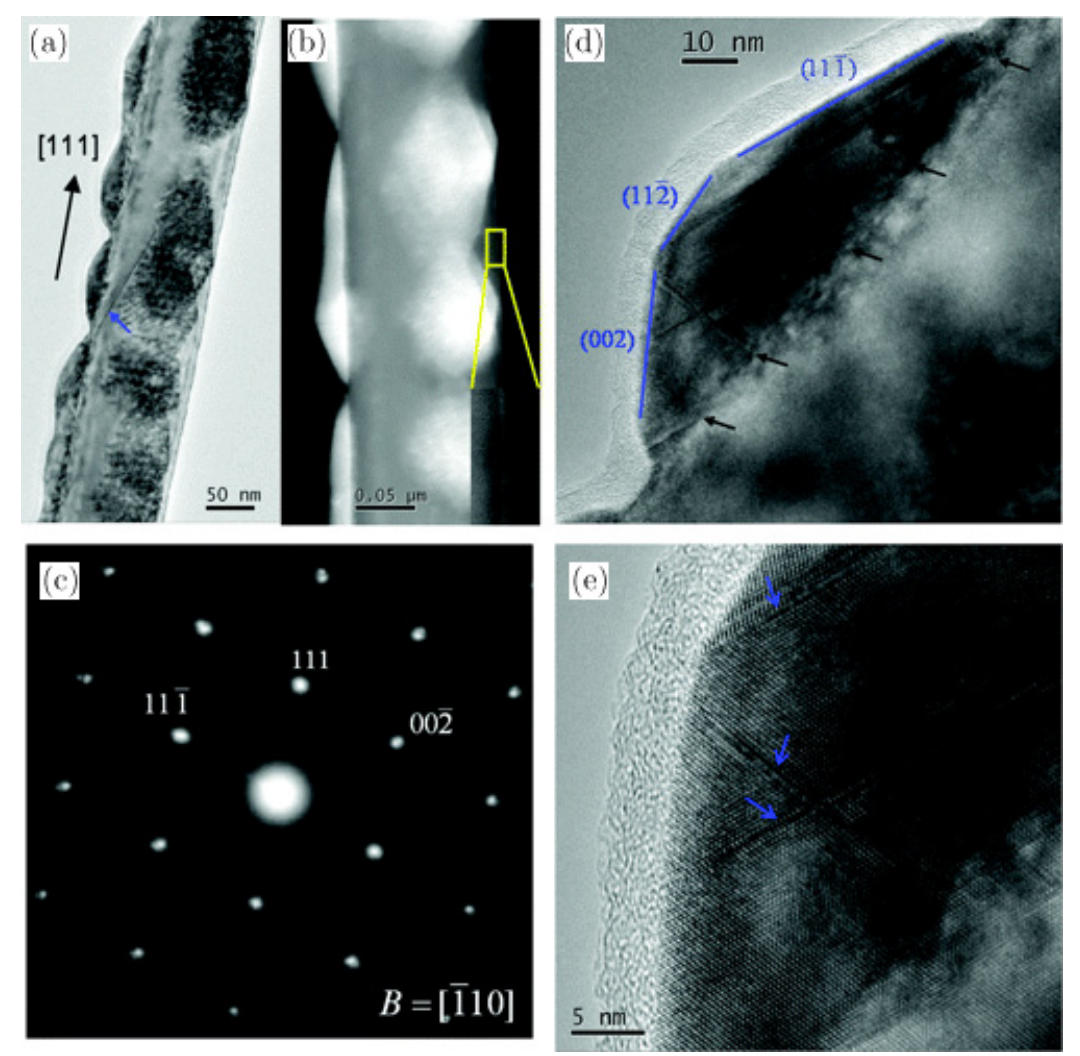

Fig. 16. (a) Bright-field TEM and (b) ADF STEM images of the Ge islands deposited on Si nanowires with Moire' fringes clearly seen. The inset in (b) shows a thin Ge-rich layer at the surface of Si nanowire. (c) The corresponding SADP with extra spots consistent with the Moire' fringes in (a) and (b). (d) Higher magnification TEM image of an island on the same nanowire with the arrows pointing to the misfit dislocations. (e) HRTEM image of an area in (d) with stacking faults and twin boundary (marked by arrows) clearly seen. Reprinted with permission from Ref. 2. Copyright 2005 Nano Letters.

Shockley partial dislocations at the core/island interface that act as misfit dislocations, as indicated by the arrow in Fig. 16(d).

In 2012, Kwon et al. ${ }^{14}$ reported the first experimental study of correlated GeQD arrays on the radial surfaces of SiNWs and their diameter dependence. Several distinctively different modes of GeQD ordering on SiNWs were observed, such as an anticorrelated pattern, single side growth and the covering of all exposed surfaces. The diameter of nanowires plays an important role in determining the morphology and distribution of GeQD arrays. In Fig. 17, periodic GeQDs arrays with an anti-correlated pattern form when the SiNW diameter is about $20 \mathrm{~nm}$. In anticorrelated patterns, if there is one quantum dot located on the right sidewall of the nanowires, no quantum dots are observed at the opposite position on the left sidewall, while quantum dots can form above or below the opposite position on the left side, as shown in Fig. 18. On the same sidewall, GeQDs are separated by a certain distance. The formation of an anti-correlated pattern is attributed to modulation of the strain field distributed within the Si core nanowire. As a GeQD is formed on the sidewall of Si core, tensile strain is created within the Si core underneath the quantum dot, and compressive strain is also produced at the opposite position within the core. The region with compressive strain is unfavorable for the growth of GeQDs. As a consequence, an anti-correlated pattern of GeQD arrays presents and decreases the total elastic energy of the system. Similarly, the effects of modulation of the strain field have also been reported in studies of multilayer quantum dot structures. In a multilayer QD sample, the strain field within the Si spacer will modulate the growth of QDs in the upper layer. ${ }^{93,94}$ However, this anticorrelated pattern disappears when the nanowire diameter is less than $15 \mathrm{~nm}$. For nanowires with a diameter of $11 \mathrm{~nm}$, GeQDs only grow on a single side of the SiNWs, as shown in Fig. 17(e), and the nanowires exhibit a bending curvature with GeQDs 

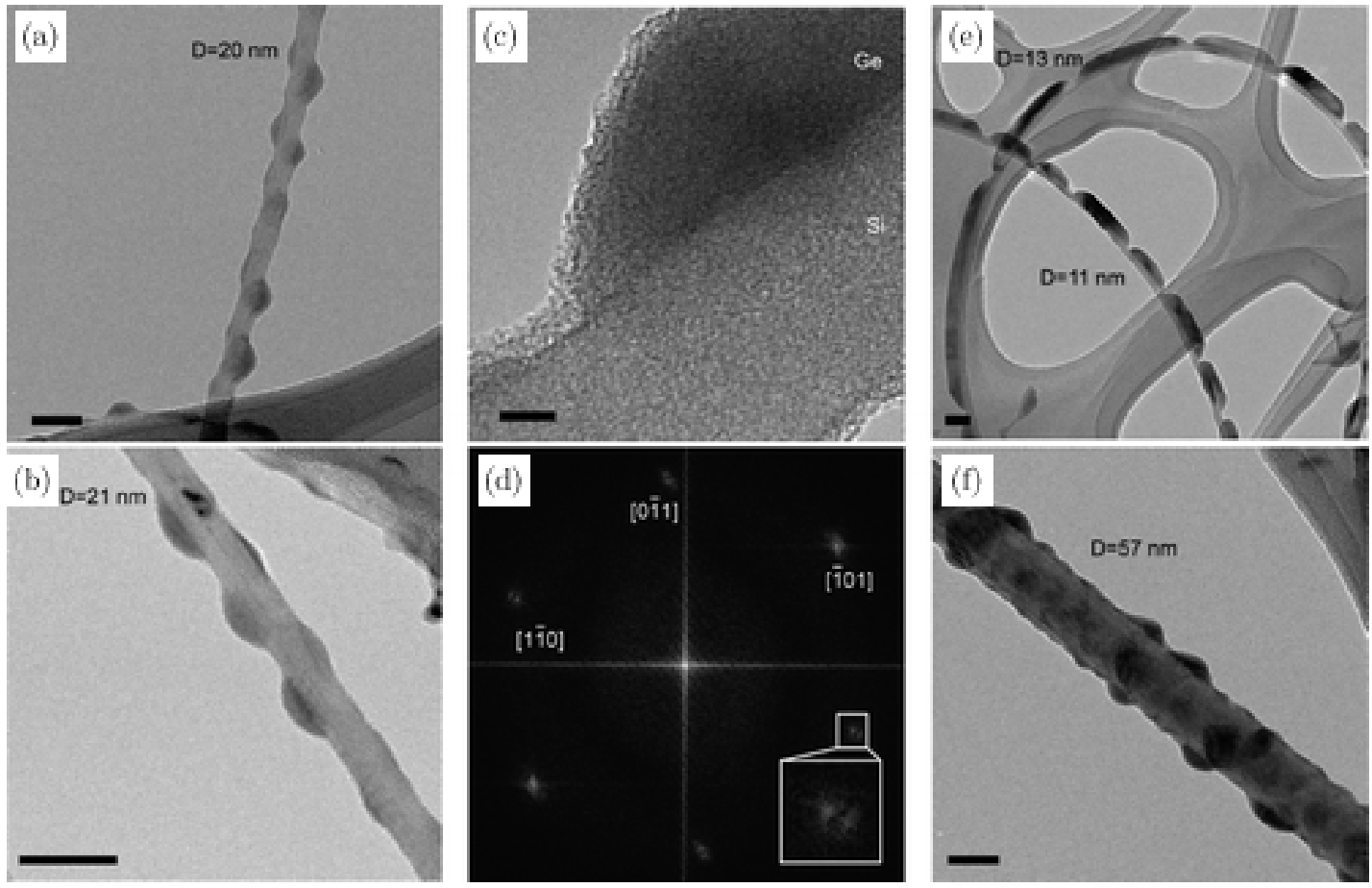

Fig. 17. TEM images of Ge QDs on Si nanowires. Both Si core and Ge QDs were grown at $470{ }^{\circ}$ C. (a) and (b) Representative TEM images of anti-correlated quantum dots with $20 \pm 2 \mathrm{~nm}$ core diameters. (c) High resolution TEM showing the defect-free single crystal Si core and Ge island. (d) FFT of the TEM image in (c). Note that the [011] and [110] FFT spots are composed of twin peak signals (lower inset). (e) and (f), morphology of Ge dots on small (11-13 nm) and large (> $50 \mathrm{~nm})$ Si cores. Scale bar, $50 \mathrm{~nm}$ for (a), (b), (d) and (e) and $5 \mathrm{~nm}$ for (c). Reprinted with permission from Ref. 14. Copyright 2012 Nano Letters.

on the convex surfaces. Such bending can be attributed to the high flexibility of this thin nanowire. In addition, when the nanowire diameter increases to $57 \mathrm{~nm}$, GeQDs are distributed randomly on all exposed surfaces of the SiNWs [Fig. 17(f)] with an arrangement and density similar to that of GeQDs on planar substrates.
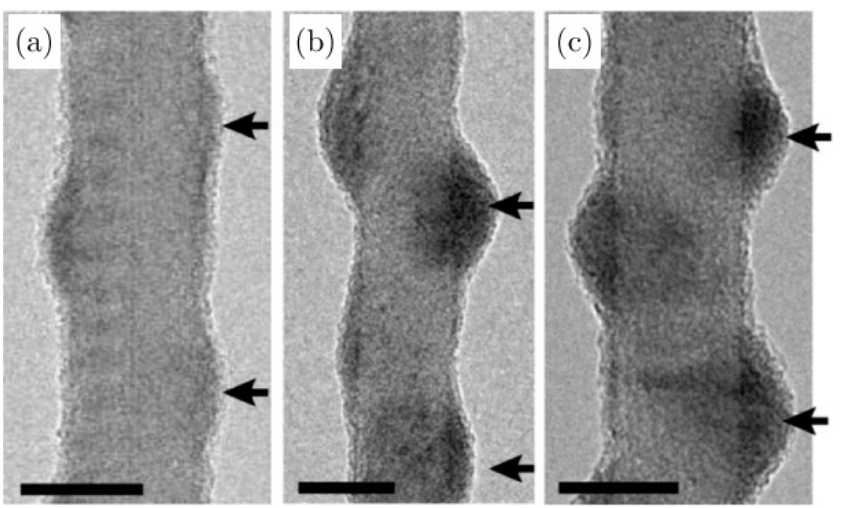

Fig. 18. Evolution of Ge QDs as a function of growth time. $(\mathrm{a}-\mathrm{c})$ TEM images showing anticorrelated array of Ge QDs at different Ge growth time; $3.5 \mathrm{~min}, 5 \mathrm{~min}$, and $10 \mathrm{~min}$ from left to right. Sale bars, $20 \mathrm{~nm}$. Reprinted with permission from Ref. 14. Copyright 2012 Nano Letters.
Pan and $\mathrm{Li}$ et al. reported that the Ge shell layer was favorable to grow in a layer-by-layer mode as $\mathrm{Si}$ nanowire diameter is less than $50 \mathrm{~nm}$. However, QDs were still observed with SiNW diameters of about $20 \mathrm{~nm}$ in Kw's experiment. These inconsistent results suggest that the growth mechanism of $\mathrm{Ge}$ shell on the radial surfaces of SiNWs needs further exploration.

In the above works, both Ge thin films and SiNWs were prepared by VLS method. Possible contamination by noble metals may affect the growth of Ge shell on the sidewalls of nanowires. Thus, Ye et $a l^{95}$ also studied the structure of GeQDs grown on the radial surfaces of SiNW by combining MACE and CVD method, instead of VLS. The diameters of SiNWs ranged between $50 \mathrm{~nm}$ and $200 \mathrm{~nm}$, and the diameters and heights of GeQDs were $40-70 \mathrm{~nm}$ and $14-20 \mathrm{~nm}$, respectively. The GeQDs were distributed randomly on all exposed surfaces of the SiNWs. The size and density of the GeQDs could be controlled effectively by changing the growth parameters. A red shift of the $\mathrm{Ge}-\mathrm{Ge}$ and $\mathrm{Si}-\mathrm{Si}$ peaks in Raman spectra was investigated due to the stress and thermal effects. 
Generally, a large surface defect density on SiNWs is produced by using MACE method, which is adverse to the growth of GeQDs on the sidewall of SiNWs and the potential application of SiNWs in photoelectronic devices. Therefore, surface defect density should be eliminated as much as possible. Before loading SiNWs into the vacuum chamber for the further growth of GeQDs, Ye et al. ${ }^{95}$ adopted the polishing solution and standard Radio Corporation of America (RCA) method to eliminate surface defects and make the surface of SiNW sidewall smooth. In addition to RCA chemical cleaning, Wu et $a .^{52}$ also grew a thicker Si buffer layer on the sidewall of SiNW to improve the growth of Ge shell layer.

In 2015, the growth of Ge particles on ordered SiNW arrays was reported by Pan et al. ${ }^{11}$ They, firstly, fabricated the ordered SiNW arrays by combining MACE and NSL technique, and then deposited a polycrystalline layer of Ge on the surface of SiNWs using electron-beam vapor. The TEM image [Fig. 19(h)] showed that Ge particles were randomly distributed on the sidewall of SiNW. The improvement of thermoelectric efficiencies has been proved after Ge particles doping on SiNWs.

However, the growth modulation of GeQDs on ordered SiNW arrays still needs to be further

explored to study their photoelectric properties. The $\mathrm{PL}$ efficiency of $\mathrm{Si} / \mathrm{Ge}$ core-shell nanowires in ordered arrays can be obviously improved by the optical resonance coupling effect (as demonstrated by $\mathrm{Wu}$ et $a l .{ }^{52}$ ), which suggests that ordered coaxial SiNW/GeQD composites have great development potential.

\subsection{Axial SiNW/GeQD composite materials}

In addition to coaxial SiNW/GeQD composite structure, the fabrication of axial SiNW/GeQD composite structure is another important research direction. As we know, SiNWs are a type of onedimensional material. Therefore, the movement of current carriers on the radial direction of nanowires is restricted while their movement along the axial direction is free. When the thickness of axial heteroepitaxial materials on nanowires is designed to be relatively small, the axial movement of current carriers is also restricted. In this case, the movement of current carriers for heteroepitaxial materials will be restricted in three dimensions, even if no islands can be formed on the axial surfaces of nanowires. This structure can also be defined as axial quantum dot/nanowire composite.

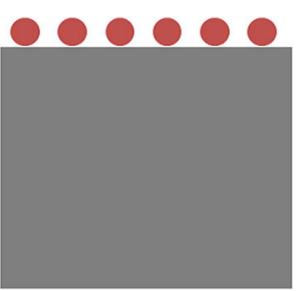

(c)

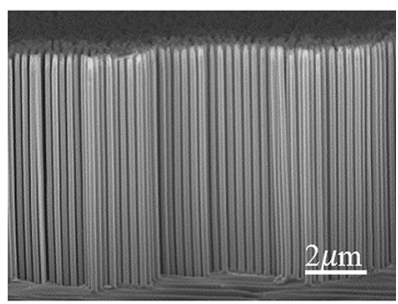

(f)

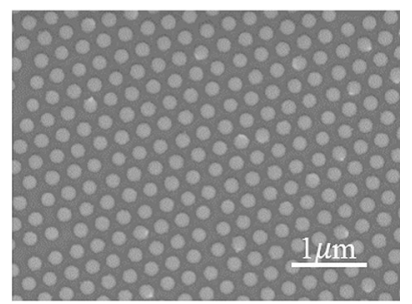

(d)

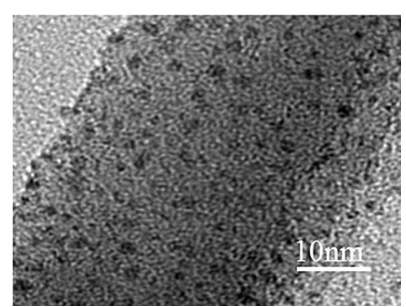

(h)

Fig. 19. Fabrication process of Ge-doped SiNWs and the corresponding SEM and TEM images. (a) and (b) Spin-coating of the PS beads (red spheres) on a silicon substrate. (c) and (d) Etching of the PS beads by isotropic RIE. (e) Gold (short yellow lines) assisted chemical etching in HF and H2O2 solution. (f) SEM image of the standing SiNWs. (g) Deposition of Ge nanoparticles (black spots) on SiNW. (h) TEM image of the lattice structure of the nanowires. The black spots are Ge particles (color online). Reprinted with permission from Ref. 11. Copyright 2015 Applied Physics Letters. 
In 2009, axial SiNW/GeQD composite structure was reported by Wen et al. ${ }^{96}$ In order to avoid the interface diffuseness between $\mathrm{Si}$ and Ge using VLS mechanism, they synthesized the axial SiNW/ GeQD composite structure by using the VSS growth mechanism. Because the VSS growth is impractically slow for conventional Si and Ge reactants, they adopted $\mathrm{AlAu}$ alloy catalyst to raise the growth rate

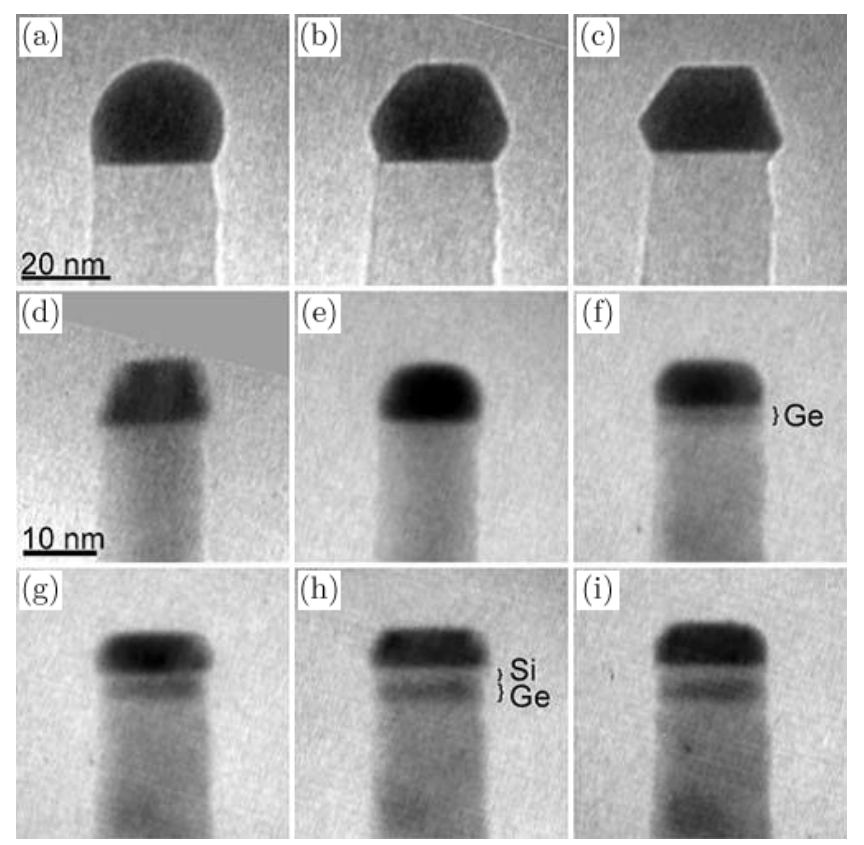

Fig. 20. (a to c) Frames extracted from a movie recorded during growth of a Si nanowire (diameter $28 \mathrm{~nm}$ ) in $3 \times 10^{-5}$ torr disilane as the temperature was reduced from $570^{\circ} \mathrm{C}$ to $503^{\circ} \mathrm{C}$ : (a) immediately before solidification of the catalyst $\left(507^{\circ} \mathrm{C}\right)$; (b) just after solidification $\left(503^{\circ} \mathrm{C}\right)$, which is evident from the faceted shape of the catalyst; (c) $14 \mathrm{~s}$ after (b), growth rate $5 \mathrm{~nm} / \mathrm{min}$. The growth rate is $22 \mathrm{~nm} / \mathrm{min}$ in the VLS mode at $570^{\circ} \mathrm{C}$. (d to i) Formation of a Si-Ge-Si heterojunction in a $\mathrm{Si}$ nanowire (diameter $14 \mathrm{~nm}$ ). The Si nanowire in (d) was first grown at $510^{\circ} \mathrm{C}$ with $1 \times 10^{-5}$ torr $\mathrm{Si}_{2} \mathrm{H}_{6}$ for $2 \mathrm{~h}$ and then cooled to $360^{\circ} \mathrm{C}$. The faceted surface after cooling indicates the solid state of the catalyst. (e) After flowing $5 \times 10^{-6}$ torr $\mathrm{Ge}_{2} \mathrm{H}_{6}$ at $360^{\circ} \mathrm{C}$ for $1 \mathrm{~min}, \mathrm{Ge}$ growth has begun and the catalyst has become less sharply faceted. (F) After $7 \mathrm{~min}$, formation of a thin Ge layer that appears as a dark band at the Si-catalyst interface. (g) After $8.5 \mathrm{~min}$ of Si growth at $360^{\circ} \mathrm{C}$ and $1 \times 10^{-5}$ torr $\mathrm{Si}_{2} \mathrm{H}_{6}$. (h) After $14 \mathrm{~min}$ of $\mathrm{Si}$ growth, the catalyst has resumed the strong facets on its surface seen in (d). (i) For demonstration purposes, the catalyst was exposed to $5 \times 10^{-6}$ torr $\mathrm{Ge}_{2} \mathrm{H}_{6}$ at $360^{\circ} \mathrm{C}$ for $1 \mathrm{~min}$ again, resuming the shape seen in (f). The growth rates of the Ge and $\mathrm{Si}$ segments measured in $(\mathrm{h})$ are 0.4 and $0.2 \mathrm{~nm} / \mathrm{min}$, respectively. The growth direction of the wires is $\mathrm{Si}[111]$. The dark contrast on the $\mathrm{Si}$ segment in (g) to (i) is due to conformal deposition of $\mathrm{Ge}$ on the $\mathrm{Si}$ sidewalls. Reprinted with permission from Ref. 96. Copyright 2009 Science. of Si and Ge. Firstly, they grew long SiNWs in the VLS mode due to its larger growth rate. Then, a Si$\mathrm{Ge}-\mathrm{Si}$ heterostructure is formed by VSS growth mechanism after cooling the growth temperature below eutectic off temperature. The results showed that the interface between GeQD and Si layer was compositional abruptness. The diameter of SiNWs is $14-28 \mathrm{~m}$, and the height of GeQD is about $3 \mathrm{~nm}$, as shown in Fig. 20. Based on their results, axial SiNW/GeQD superlattice structures are expected to be developed. The structure is similar to an axial $\mathrm{Ge} / \mathrm{Si}$ superlattice nanowire structure, ${ }^{97,98}$ but the thickness of the Ge layer in an axial SiNW/GeQD structure is lower than that in an axial $\mathrm{Ge} / \mathrm{Si}$ superlattice nanowire structure.

Since ordered nanowire arrays can form optical whispering gallery microcavities (WGMs), the quantum efficiency of QDs can be improved significantly by embedding them into nanowires. ${ }^{99-101}$ Therefore, the growth of GeQDs on the top surface of larger SiNWs has been studied recently. Zhou et $a l^{102}$ investigated the growth of GeQDs on the tops of periodically arranged SiNWs with diameters of 350-450 nm. As shown in Fig. 21, for nanowires with a diameter of $450 \mathrm{~nm}$, four small hut-like GeQDs are symmetrically distributed at the edges of the top terraces of the SiNWs. The QDs have heights of $13.3 \pm 0.9 \mathrm{~nm}$ and sidewalls with $\{105\}$ facets. This is attributed to local potential minima appearing at the corners of the tops of SiNWs with larger diameters, where Ge atoms will be in favor of nucleation. For nanowires with a diameter of $350 \mathrm{~nm}$, one large dome-like QD is located at the center of the top terrace of the nanowire. This indicates that the SiNW diameter determines the distribution and morphology of the GeQDs. After

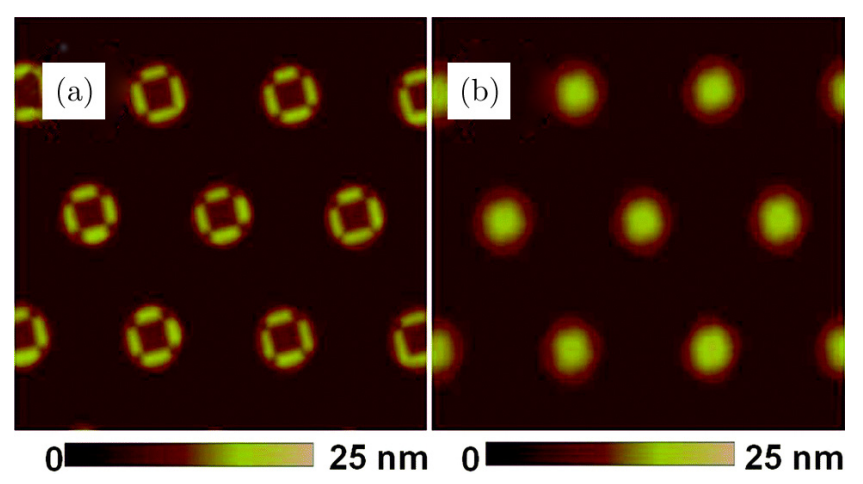

Fig. 21. AFM images of the surface morphologies after $10 \mathrm{ML}$ Ge deposition on SMPs of (a) $\rho_{600}^{450}$ and (b) $\rho_{450}^{350}$ at $580^{\circ} \mathrm{C}$. Reprinted with permission from Ref. 102. Copyright 2014 Nanoscale. 

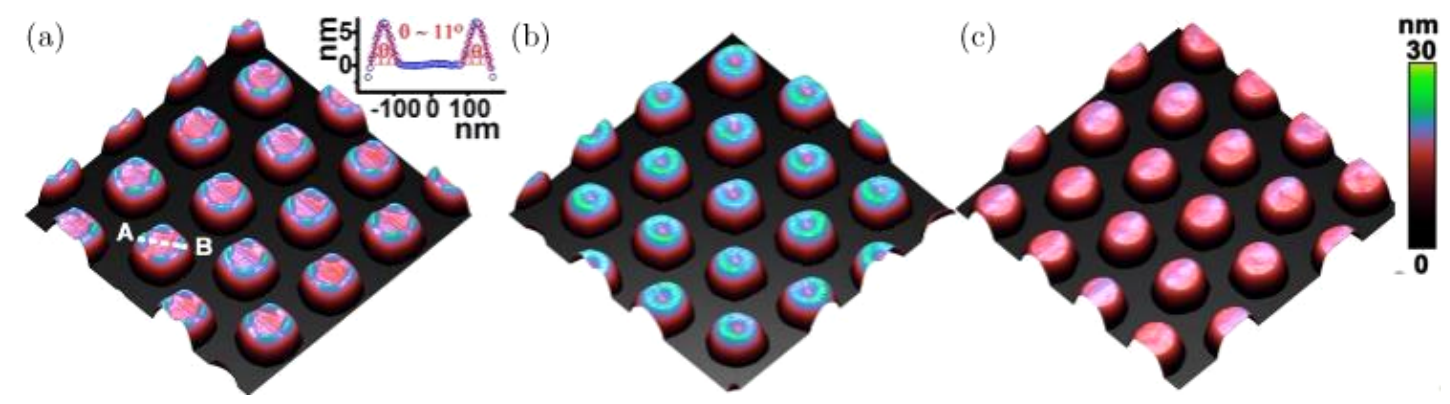

Fig. 22. AFM images $\left(2 \times 2 \mu \mathrm{m}^{2}\right)$ of self-assembled Ge nanostructures on the ordered Si nano-pillars via the two-step procedure with the Ge deposition of, (a) $\left(0.8 \mathrm{~nm}\right.$ at $500^{\circ} \mathrm{C}$ and $\left.0.1 \AA / \mathrm{s}\right)+\left(1.0 \mathrm{~nm}\right.$ at $520^{\circ} \mathrm{C}$ and $\left.0.025 \AA / \mathrm{s}\right),(\mathrm{b})\left(0.8 \mathrm{~nm}\right.$ at $500^{\circ} \mathrm{C}$ and $\left.0.1 \AA / \mathrm{s}\right)$ $+\left(1.0 \mathrm{~nm}\right.$ at $580^{\circ} \mathrm{C}$ and $\left.0.025 \AA / \mathrm{s}\right)$, (c) $\left(0.8 \mathrm{~nm}\right.$ at $580^{\circ} \mathrm{C}$ and $\left.0.025 \AA / \mathrm{s}\right)+\left(1.0 \mathrm{~nm}\right.$ at $480^{\circ} \mathrm{C}$ and $\left.0.1 \AA / \mathrm{s}\right)$. The inset in (a) shows the height profile along the dotted line $\mathrm{AB}$ in (a). The color bar is shown at the right (color online). Reprinted with permission from Ref. 103. Copyright 2016 Scientific Reports.

embedding QDs at the edges of the top terrace and close to the sidewalls of the nanowires, strong lightmatter coupling phenomena such as resonance radiation and Purcell effects are expected if the spontaneous radiation of quantum dots can match the WGM resonance mode. Wang et al. ${ }^{103}$ proposed a two-step growth method to control the uniform growth of $\mathrm{Ge}$ atoms at the edges of the tops of SiNWs. In their experiment, $0.8 \mathrm{~nm}$ of Ge was first deposited at $500^{\circ} \mathrm{C}$ at a rate of $0.1 \AA / \mathrm{s}$. After the growth was interrupted for $1 \mathrm{~min}$, another $1 \mathrm{~nm}$ of Ge was deposited at different temperatures at a rate of $0.025 \AA /$ s. In Fig. 22, when the temperature of the second $\mathrm{Ge}$ growth was $500^{\circ} \mathrm{C}$, four uniform QDs were formed at each edge of the top terrace. With the temperature increased to $580^{\circ} \mathrm{C}$, a quantum ring was acquired at the top of the nanowire. With the temperature decreased to $480^{\circ} \mathrm{C}$, no quantum dots or quantum ring occurred.

\section{Conclusions}

In this review, recent research progress on the fabrication and structures of SiNWs, GeQDs and SiNW/GeQD composites was introduced, respectively. Firstly, ordered nanowire arrays with small diameters, large aspect ratios and special structures become the hot research topics. Then, the researches about GeQDs focus on improving their luminescence properties by designing novel structures, such as GeQD crystals, Ge/Si/Ge CQD, and GIBGeQDs. In experimental studies of coaxial SiNW/ GeQD composites, there are inconsistent conclusions regarding the critical SiNW diameter for the transition of Ge growth from the F-M to the S-K mode. Simulations show that growth of Ge thin films on SiNWs is more inclined to form Si/Ge coreshell nanowires as the nanowire diameter becomes smaller, whereas it is easier to prepare SiNW/GeQD composite structures with larger diameter nanowires. A physical mechanism has been put forward to understand the transition of Ge growth mode on SiNWs with increasing nanowire diameter. It has also been found that the curved surfaces of SiNWs help to release the strain induced by lattice mismatch. Furthermore, the axial SiNW/GeQD composite structure have already been demonstrated by VSS method. For the potential application, axial SiNW/GeQD superlattice structures are expected to be developed. In summary, the studies about SiNW/GeQD composites have raised a lot of interesting questions, which need to be resolved in future. By reviewing the research on the preparation methods and structures of SiNW/GeQD composite materials, we hope to facilitate more intensive study of such materials and their potential applications.

\section{Acknowledgments}

This work was supported by the National Natural Science Foundation of China (Nos. 11504322, 11564043, 11804295 and 11704330), and the key Project of Applied Basic Research Program of Yunnan Province of China (Nos. 2016FB002 and 2016FB006). Jie Yang also appreciates the support from Yunnan University by the Project of Training for Dong Lu Young Teachers.

\section{References}

1. X. Chen, Q. Bi, M. Sajjad, X. Wang, Y. Ren, X. Zhou, W. Xu and Z. Liu, Nanomaterials 8, 285 (2018). 
2. L. Pan, K. K. Lew, J. M. Redwing and E. C. Dickey, Nano Lett. 5, 1081 (2005).

3. A. K. Katiyar, A. K. Sinha, S. Manna and S. K. Ray, ACS Appl. Mater. Interfaces 6, 15007 (2014).

4. G. Z. Chen, M. S. P. Shaffer, D. Coleby, G. Dixon, W. Zhou, D. J. Fray and A. H. Windle, Adv. Mater. 12, $522(2000)$.

5. K. Un Jeong, L. Eun Hong, K. Jong Min, M. Yo-Sep, K. Eunseong and P. Wanjun, Nanotechnology 28, 295201 (2009).

6. M. S. Gudiksen, L. J. Lauhon, J. Wang, D. C. Smith and C. M. Lieber, Nature 415, 617 (2002).

7. C. J. Kim, H. S. Lee, Y. J. Cho, J. E. Yang, R. R. Lee, J. K. Lee and M. H. Jo, Adv. Mater. 23, 1025 (2015).

8. S. Manna, S. Das, S. P. Mondal, R. Singha and S. K. Ray, J. Phys. Chem. C 116, 7126 (2012).

9. Z. Liu, T. Zhou, L. Li, Y. Zuo, C. He, C. Li, C. Xue, B. Cheng and Q. Wang, Appl. Phys. Lett. 103, 4117 (2013).

10. Y. L. Chang, J. L. Wang, F. Li and Z. Mi, Appl. Phys. Lett. 96, 4741 (2010).

11. Y. Pan, G. Hong, S. N. Raja, S. Zimmermann, M. K. Tiwari and D. Poulikakos, Appl. Phys. Lett. 106, 093102 (2015).

12. N. Fukata, M. Mitome, T. Sekiguchi, Y. Bando, M. Kirkham, J. I. Hong, Z. L. Wang and R. L. Snyder, ACS Nano 6, 8887 (2012).

13. X. Li and G. Yang, Nanotechnology 25, 435605 (2014).

14. S. Kwon, Z. C. Chen, J. H. Kim and J. Xiang, Nano Lett. 12, 4757 (2012).

15. K. B. Dhungana, M. Jaishi and R. Pati, Nano Lett. 16, 3995 (2016).

16. R. S. Wagner and W. C. Ellis, Appl. Phys. Lett. 4, 89 (1964).

17. Y. F. Zhang, Y. H. Tang, N. Wang, D. P. Yu, C. S. Lee, I. Bello and S. T. Lee, Appl. Phys. Lett. 72, 1835 (1998).

18. Z. Huang, Nano Lett. 8, 3046 (2008).

19. F. C. K. Au, K. W. Wong, Y. H. Tang, Y. F. Zhang, I. Bello and S. T. Lee, Appl. Phys. Lett. 75, 1700 (1999).

20. N. J. Stone and H. Ahmed, Appl. Phys. Lett. 73, 2134 (1998).

21. S. F. Hu, W. Z. Wong, S. S. Liu, Y. G. Wu, C. L. Sung, T. Y. Huang and S. M. Sze, Solid State Commun. 125, 351 (2003).

22. J. D. Holmes, K. P. Johnston, R. C. Doty and B. A. Korgel, Science 287, 1471 (2000).

23. Y. Zhao, J. Yu, L. G. Fang, J. Zheng, H. Q. Wang, J. R. Yuan, S. Wu and G. A. Cheng, Nanoscale Res. Lett. 10, 1 (2015).

24. C. J. Kim, H. S. Lee, Y. J. Cho, J. E. Yang, R. R. Lee, J. K. Lee and M. H. Jo, Adv. Mater. 23, 1025 (2015).
25. A. M. Morales and C. M. Lieber, Science 279, 208 (1998).

26. Y. Wu, Y. Cui, L. Huynh, C. J. Barrelet, D. C. Bell and C. M. Lieber, Nano Lett. 4, 433 (2004).

27. D. J. Hill, T. S. Teitsworth, S. Kim, J. D. Christesen and J. F. Cahoon, ACS Appl. Mater. Interfaces 9, 37105 (2017).

28. B. Fuhrmann, H. S. Leipner, H. R. Höche, L. Schubert, P. Werner and U. Gösele, Nano Lett. 5, 2524 (2005).

29. Y. S. Park and J. S. Lee, Chem. Asian J. 11, 1878 (2016).

30. A. I. Hochbaum, R. Chen, R. D. Delgado, W. Liang, E. C. Garnett, M. Najarian, A. Majumdar and P. Yang, Nature 451, 163 (2008).

31. A. I. Hochbaum, D. Gargas, Y. J. Hwang and P. Yang, Nano Lett. 9, 3550 (2009).

32. Y. J. Hung, S. L. Lee, K. C. Wu, Y. Tai and Y. T. Pan, Opt. Exp. 19, 15792 (2011).

33. Z. Tan, W. Shi, C. Guo, Q. Zhang, L. Yang, X. Wu, G. A. Cheng and R. Zheng, Nanoscale 7, 17268 (2015).

34. Z. Huang, H. Fang and J. Zhu, Adv. Mater. 19, 744 (2007).

35. S. W. Chang, V. P. Chuang, S. T. Boles, C. A. Ross and C. V. Thompson, Adv. Func. Mater. 19, 2495 (2009).

36. K. Balasundaram, J. S. Sadhu, J. C. Shin, B. Azeredo, D. Chanda, M. Malik, K. Hsu, J. A. Rogers, P. Ferreira and S. Sinha, Nanotechnology 3, 305304 (2012).

37. J. Kim, H. Han, Y. H. Kim, S. H. Choi, J. C. Kim and W. Lee, ACS Nano 5, 3222 (2011).

38. L. Li, Y. Fang, C. Xu, Y. Zhao, N. Zang, P. Jiang and K. J. Ziegler, Nanotechnology 27, 165303 (2016).

39. L. Li, Y. Fang, C. Xu, Y. Zhao, K. Wu, C. Limburg, P. Jiang and K. J. Ziegler, ACS Appl. Mater. Interfaces 9, 7368 (2017).

40. Y. Chen, L. Li, C. Zhang, C. C. Tuan, X. Chen, J. Gao and C. P. Wong, Nano Lett. 17, 1014 (2017).

41. Y. Chen, C. Zhang, L. Li, C. C. Tuan, F. Wu, X. Chen, J. Gao, Y. Ding and C. P. Wong, Nano Lett. 17, 4304 (2017).

42. V. Schmidt, J. V. Wittemann and U. Gosele, Chem. Rev. 110, 361 (2010).

43. M. Amato, M. Palummo, R. Rurali and S. Ossicini, Chem. Rev. 114, 1371 (2014).

44. V. Schmidt, J. V. Wittemann and U. Gösele, Chem. Rev. 110, 361 (2010).

45. M. Zamfir, H. Nguyen, E. Moyen, Y. Lee and D. Pribat, J. Mater. Chem. A 1, 9566 (2013).

46. A. N. Goldstein and A. P. Alivisatos, Science 256, 1425 (1992). 
47. P. Gentile, A. Solanki, N. Pauc, F. Oehler, B. Salem, G. Rosaz, T. Baron, M. D. Hertog and V. Calvo, Nanotechnology 23, 215702 (2012).

48. S. K. Ray, A. K. Katiyar and A. K. Raychaudhuri, Nanotechnology 28, 092001 (2017).

49. D. Zhang, G. Cheng, J. Wang, C. Zhang, Z. Liu, Y. Zuo, J. Zheng, C. Xue, C. Li and B. Cheng, Nanoscale Res. Lett. 9, 661 (2014).

50. M. L. Zhang, K. Q. Peng, X. Fan, J. S. Jie, R. Q. Zhang, S. T. Lee and N. B. Wong, J. Phys. Chem. $C$ 112, 4444 (2008).

51. A. I. Hochbaum, R. Chen, R. D. Delgado, W. Liang, E. C. Garnett, M. Najarian, A. Majumdar and P. Yang, Nature 39, 163 (2008).

52. Z. Wu, H. Lei, T. Zhou, Y. Fan and Z. Zhong, Nanotechnology 25, 055204 (2014).

53. H. Han, J. Kim, H. S. Shin, J. Y. Song and W. Lee, Adv. Mater. 24, 2284 (2012).

54. B. J. De, N. Geyer, J. V. Wittemann, U. Gösele and V. Schmidt, Nanotechnology 21, 095302 (2010).

55. C. G. Chen, R. K. Heilmann, C. Joo, P. T. Konkola and G. S. Pati, J. Vac. Sci. Technol. B 203071 (2002).

56. Y. Qu, L. Liao, Y. Li, H. Zhang, Y. Huang and X. Duan, Nano Lett. 9, 4539 (2009).

57. C. L. Lee, K. Tsujino, Y. Kanda, S. Ikeda and M. Matsumura, J. Mater. Chem. 18, 1015 (2008).

58. A. Plettl, F. Enderle, M. Saitner, A. Manzke, C. Pfahler, S. Wiedemann and P. Ziemann, Adv. Func. Mater. 19, 3279 (2009).

59. J. Yang, M. Zhang, X. Lan, X. Weng, Q. Shu, R. Wang, F. Qiu, C. Wang and Y. Yang, Nanoscale Res. Lett. 13, 177 (2018)

60. D. J. Eaglesham and M. Cerullo, Phys. Rev. Lett. 64, 1943 (1990).

61. Y. W. Mo, D. E. Savage, B. S. Swartzentruber and M. G. Lagally, Mod. Phys. Lett. B 4, 22 (1990).

62. S. Pasić and K. Ilakovac, Phys. Rev. A 55, 4248 (1997).

63. D. Dovinos and D. Williams, Phys. Rev. B 72, 8 (2005).

64. R. Aluguri, S. Manna and S. K. Ray, J. Appl. Phys. 115, 013502 (2014).

65. X. L. Li, C. X. Wang and G. W. Yang, Progr. Mater. Sci. 64, 121 (2014).

66. X. L. Li, Y. Y. Cao and G. W. Yang, Phys. Chem. Chem. Phys. 12, 4768 (2010).

67. X. L. Li, J. Phys. Chem. C 114, 2018 (2010).

68. A. I. Yakimov, A. V. Dvurechenskii, A. I. Nikiforov and Y. Y. Proskuryakov, J. Appl. Phys. 89, 5676 (2001).

69. M. H. Liao, C. Y. Yu, T. H. Guo, C. H. Lin and C. W. Liu, IEEE Electron. Device Lett. 27, 252 (2006).
70. P. W. Li, W. M. Liao, D. M. T. Kuo, S. W. Lin, P. S. Chen, S. C. Lu and M. J. Tsai, Appl. Phys. Lett. 85, 1532 (2004).

71. T. Zhou and Z. Zhong, Apl. Mater. 2, 1866 (2014).

72. S. Das, K. Das, R. K. Singha, S. Manna, A. Dhar, S. K. Ray and A. K. Raychaudhuri, Nanoscale Res. Lett. 6, 416 (2011).

73. K. F. Wang, Z. Yang and W. Zhang, Appl. Surf. Sci. 258, 1935 (2012).

74. R. Aluguri, S. Manna and S. K. Ray, J. Appl. Phys. 115, 8 (2014).

75. A. B. Talochkin, A. A. Shklyaev and V. I. Mashanov, J. Appl. Phys. 115, 27 (2014).

76. Y. Chen, S. Wu, Y. Ma, Y. Fan, X. Yang, Z. Zhong and Z. Jiang, J. Appl. Phys. 116, 233502 (2014).

77. M. H. Kuo, W. T. Lai, S. W. Lee, Y. C. Chen, C. W. Chang, W. H. Chang, T. M. Hsu and P. W. Li, Opt. Lett. 40, 2401 (2015).

78. C. Dais, G. Mussler, T. Fromherz, E. Müller, H. H. Solak and D. Grützmacher, Nanotechnology 26, 255302 (2015).

79. H. Groiss, L. Spindlberger, P. Oberhumer, F. Schäffler, T. Fromherz, M. Grydlik and M. Brehm, Semicond. Sci. Technol. 32, 2 (2017).

80. V. A. Zinovyev, A. F. Zinovieva, P. A. Kuchinskaya, Z. V. Smagina, V. A. Armbrister, A. V. Dvurechenskii, O. M. Borodavchenko, V. D. Zhivulko and A. V. Mudryi, Appl. Phys. Lett. 110, 102101 (2017).

81. J. Yang, B. Zhao, C. Wang, F. Qiu, R. F. Wang and Y. Yang, Appl. Surf. Sci. 286, 303 (2016)

82. J. Yang, X. K. Weng, M. L. Zhang, T. Yang, F. Qiu, R. F. Wang, C. Wang and Y. Yang, Vacuum 154, $115(2018)$

83. X. Li and O. Gang, J. Appl. Phys. 109, 725 (2011).

84. X. L. Li, Phys. Chem. Chem. Phys. 15, 5238 (2013).

85. A. Karmous, A. Cuenat, A. Ronda, I. Berbezier, S. Atha and R. Hull, Appl. Phys. Lett. 85, 6401 (2004).

86. Y. Cui, X. Duan, A. Jiangtao Hu and C. M. Lieber, J. Phys. Chem. B 104, 5213 (2000).

87. C. J. Kim, H. S. Lee, Y. J. Cho, J. E. Yang, R. R. Lee, J. K. Lee and M. H. Jo, Adv. Mater. 23, 1025 (2011).

88. X. L. Li and G. W. Yang, Appl. Phys. Lett. 92, 171902 (2008).

89. H. Wang, M. Upmanyu and C. V. Ciobanu, Nano Lett. 8, 4305 (2008).

90. V. Schmidt, P. C. Mclntyre and U. Goesele, Phys. Rev. B 77, 235302 (2008).

91. J. L. Taraci, M. J. Hytch, T. Clement, P. Peralta and M. R. McCartney, Nanotechnology 16, 2365 (2005). 
92. J. Y. Guo, Y. W. Zhang and V. B. Shenoy, Acs Nano 4, 4455 (2010).

93. V. L. Thanh, V. Yam, P. Boucaud, F. Fortuna, C. Ulysse, D. Bouchier, L. Vervoort and J. M. Lourtioz, Phys. Rev. B Condens. Mat. 60, 5851 (1999).

94. E. Mateeva, P. Sutter, J. C. Bean and M. G. Lagally, Appl. Phys. Lett. 71, 3233 (1997).

95. M. Ye, D. Wang, Z. Xu, L. Pu, Y. Shi, M. Han, R. Zhang and Y. Zheng, J. Func. Mater. 41, 1332 (2010) (in Chinese).

96. C.-Y. Wen, M. C. Reuter, J. Bruley, J. Tersoff, S. Kodambaka, E. A. Stach and F. M. Ross, Science 326, 1247 (2009).
97. Y. Wu, R. Fan and P. Yang, Nano Lett. 2, 83 (2002).

98. Y. Jiang, S. Huang, Z. Zhu, C. Zeng, Y. Fan and Z. Jiang, Nanoscale Res. Lett. 11, 102 (2016).

99. K. J. Vahala, Nature 424, 839 (2003).

100. C. Bruggemann, A. V. Akimov, A. V. Scherbakov, M. Bombeck, C. Schneider, S. Hofling, A. Forchel, D. R. Yakovlev and M. Bayer, Nat. Photon. 6, 30 (2012).

101. J. S. Xia, Y. Ikegami, Y. Shiraki, N. Usami and Y. Nakata, Appl. Phys. Lett. 89, 3509 (2006).

102. T. Zhou, C. Zeng, Q. Ma, Y. Ma, Y. Fan, Z. Jiang, J. Xia and Z. Zhong, Nanoscale 6, 3925 (2014).

103. S. Wang, T. Zhou, D. Li and Z. Zhong, Sci. Rep. 6, 28872 (2016). 